\title{
Topology optimization of two-dimensional elastic wave barriers
}

\author{
C. Van hoorickx ${ }^{\mathrm{a}, *}$, O. Sigmund ${ }^{\mathrm{b}}$, M. Schevenels ${ }^{\mathrm{c}}$, B.S. Lazarov ${ }^{\mathrm{b}}$, G. Lombaert ${ }^{\mathrm{a}}$ \\ ${ }^{a} K U$ Leuven, Department of Civil Engineering, Structural Mechanics Section, \\ Kasteelpark Arenberg 40, 3001 Leuven, Belgium \\ ${ }^{b}$ Technical University of Denmark, Department of Mechanical Engineering, Solid Mechanics, \\ Nils Koppels Allé, Building 404, 2800 Lyngby, Denmark \\ ${ }^{c} K U$ Leuven, Department of Architecture, Architectural Engineering, \\ Kasteelpark Arenberg 1, 3001 Leuven, Belgium
}

\begin{abstract}
Topology optimization is a method that optimally distributes material in a given design domain. In this paper, topology optimization is used to design two-dimensional wave barriers embedded in an elastic halfspace. First, harmonic vibration sources are considered, and stiffened material is inserted into a design domain situated between the source and the receiver to minimize wave transmission. At low frequencies, the stiffened material reflects and guides waves away from the surface. At high frequencies, destructive interference is obtained that leads to high values of the insertion loss. To handle harmonic sources at a frequency in a given range, a uniform reduction of the response over a frequency range is pursued. The minimal insertion loss over the frequency range of interest is maximized. The resulting design contains features at depth leading to a reduction of the insertion loss at the lowest frequencies and features close to the surface leading to a reduction at the highest frequencies. For broadband sources, the average insertion loss in a frequency range is optimized. This leads to designs that especially reduce the response at high frequencies. The designs optimized for the frequency averaged insertion loss are found to be sensitive to geometric imperfections. In order to obtain a robust design, a worst case approach is followed.
\end{abstract}

Keywords: Topology optimization, Elastodynamic wave propagation, Vibration reduction measures, Wave barriers

\section{Introduction}

This paper focuses on reducing two-dimensional wave transmission in an elastic halfspace by designing a wave barrier using topology optimization. Topology optimization searches for the most efficient distribution of a given amount of material in a specified design domain [1]. The problem is formulated as a mathematical optimization problem where the performance of the structure is maximized while satisfying a set of constraints such as the volume fraction of the stiffened material. Topology optimization simultaneously optimizes not only the size and the shape of the design, but also the topology, making it possible to obtain novel, improved design geometries.

Topology optimization was originally developed for static mechanical problems, but has since then been used for a variety of applications including problems governed by wave propagation [2]. A lot of research has been performed in the field of photonic crystal waveguide design. These electromagnetic waveguides are designed based on the band-gap phenomenon in periodic structures, obstructing wave propagation for specific frequencies $[3,4]$.

\footnotetext{
*Corresponding author. Tel.: +321632 2204

Email addresses: cedric.vanhoorickx@bwk.kuleuven.be (C. Van hoorickx), sigmund@mek.dtu.dk (O. Sigmund), mattias.schevenels@asro.kuleuven.be (M. Schevenels), bsl@mek.dtu.dk (B.S. Lazarov), geert.lombaert@bwk.kuleuven.be (G. Lombaert)

Postprint submitted to Journal of Sound and Vibration

Published version: C. Van hoorickx, O. Sigmund, M. Schevenels, B.S. Lazarov, and G. Lombaert. ogy optimization of two-dimensional elastic wave barriers. Journal of Sound and Vibration, 376:95-111, 2016. http://dx.doi.org/10.1016/j.jsv.2016.04.039
} 
Next to photonic band-gaps due to electromagnetic waves, also phononic band-gaps due to elastic and acoustic waves have been investigated. Sigmund and Jensen [5] maximize the bandwidth of phononic bandgap materials and minimize the transmitted wave amplitude of a band-gap structure subjected to harmonic loading. For a one-dimensional homogeneous elastodynamic medium, topology optimization leads to periodic structures where the thicknesses of the layers are equal to a quarter of the considered wavelength [6]. Jensen [7] discusses a two-dimensional problem, where an incoming plane pressure or shear wave is maximally reflected or dissipated by an optimal periodic distribution of scattering or absorbing inclusions.

Structural optimization has also been applied to acoustic design problems. Wadbro and Berggren [8] optimize an acoustic horn that efficiently radiates sound. Duhring et al. [9] use topology optimization for two types of problems. First, a room acoustic problem is considered where reflecting material is optimally distributed along the ceiling or the walls. Second, an outdoor sound barrier is optimized to reduce the sound power level behind the barrier. The same problem was considered by Greiner et al. [10] who applied shape optimization using genetic algorithms to optimize Y-shaped noise barriers. Shape optimization is also used by Abe et al. [11] to optimize noise barriers for railway viaducts and topology optimization is used by Kook et al. [12] to minimize the maximum main specific loudness.

Structural optimization is often found to lead to designs which are very sensitive to geometrical imperfections [13]. Since small variations in the design might result in a strongly deteriorated performance, robust optimization methods have been developed to take these uncertainties into account. Sigmund [14] and Wang et al. [15] proposed a robust optimization method to deal with manufacturing tolerances. A projection filter is added to the optimization to ensure a black and white design. The projection threshold is varied to simulate geometric imperfections and the worst performance of multiple designs originating from different projection thresholds is optimized. This methodology is applied to the elastodynamic problem in this paper to obtain designs that are both effective and robust with respect to geometrical imperfections.

This paper investigates the use of topology optimization for the design of two-dimensional wave barriers impeding wave transmission between a source and receiver. This problem is encountered in problems of environmental vibrations, e.g. as generated by railway traffic [16]. The finite element method is used to determine the displacement field in the halfspace, with Perfectly Matched Layers (PMLs) at the boundaries to prevent spurious reflections. In order to minimize the transmission of waves, stiffened material is distributed in a design domain situated between the source and the receiver. A gradient-based optimization method is applied and the sensitivities are calculated using the adjoint method, making the calculation efficient for the large number of design variables considered. A worst case approach is adopted for obtaining a robust design with respect to geometrical imperfections where the worst performance of some (extreme) cases is optimized. This paper demonstrates the importance of considering robustness in the optimization process, and shows that the robust designs can be utilized as a source for simplified design solutions.

This paper is organized as follows. First, the method of topology optimization is briefly recapitulated and the optimization problem is presented. Next, wave barriers are designed with topology optimization for three types of excitation: harmonic point sources at a known frequency, harmonic point sources at a frequency situated in a given range, and broadband point sources. Finally, the sensitivity of the optimized design with respect to geometric imperfections is investigated and robust topology optimization is applied to obtain designs less sensitive to these uncertainties.

\section{Formulation of the optimization problem}

\subsection{Finite element model}

Figure 1 shows the considered optimization problem. A two-dimensional homogeneous elastic halfspace of material 1 is excited at the surface by a vertical point load. The aim is to minimize the response at an output point, located at the surface of the halfspace. Therefore, a design domain is considered between the source and the receiver where a second stiffer material is introduced. The design domain has a crosssectional area of $5 \times 8 \mathrm{~m}^{2}$ and is located at a distance of $5 \mathrm{~m}$ from the excitation point. The position where the performance is optimized is at another $5 \mathrm{~m}$ from the design domain, and, therefore, $15 \mathrm{~m}$ from the excitation point. The properties of the original material 1 and the stiffened material 2 are summarized in table 1, with 
$\rho$ the mass density, $C_{\mathrm{p}}$ the longitudinal wave velocity, $C_{\mathrm{s}}$ the shear wave velocity, $\lambda_{\mathrm{p}}$ the wavelength of the longitudinal waves and $\lambda_{\mathrm{s}}$ the wavelength of the shear waves.

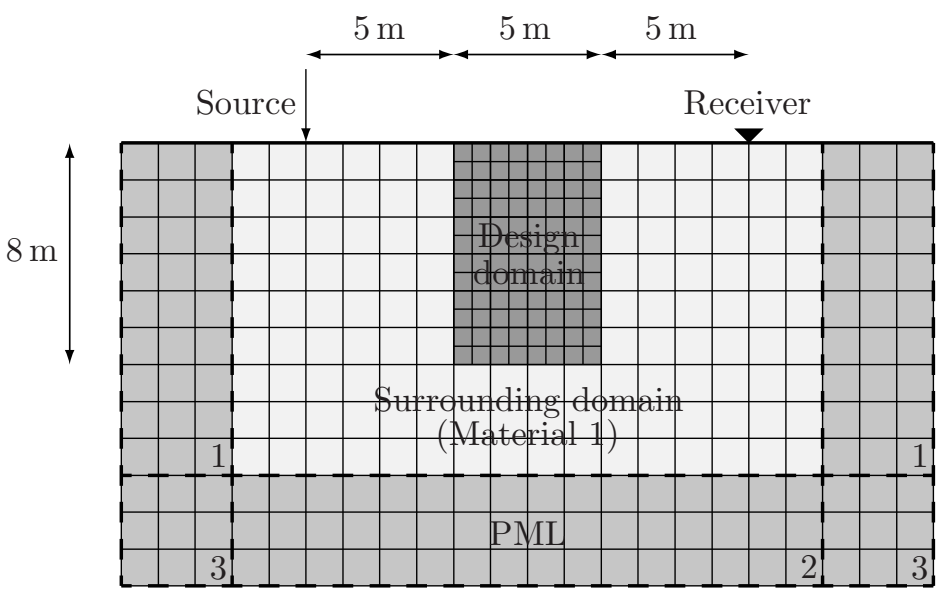

Figure 1: The considered two-dimensional topology optimization problem.

Table 1: Material properties in the optimization problem and corresponding longitudinal and shear wavelengths at two frequencies.

\begin{tabular}{c|cc}
\hline Property & Material 1 & Material 2 \\
\hline$\rho$ & $2000 \mathrm{~kg} / \mathrm{m}^{3}$ & $2000 \mathrm{~kg} / \mathrm{m}^{3}$ \\
$C_{\mathrm{p}}$ & $400 \mathrm{~m} / \mathrm{s}$ & $950 \mathrm{~m} / \mathrm{s}$ \\
$C_{\mathrm{s}}$ & $200 \mathrm{~m} / \mathrm{s}$ & $550 \mathrm{~m} / \mathrm{s}$ \\
\hline$\lambda_{\mathrm{p}, 25 \mathrm{~Hz}}$ & $16 \mathrm{~m}$ & $38 \mathrm{~m}$ \\
$\lambda_{\mathrm{s}, 25 \mathrm{~Hz}}$ & $8 \mathrm{~m}$ & $22 \mathrm{~m}$ \\
$\lambda_{\mathrm{p}, 50 \mathrm{~Hz}}$ & $8 \mathrm{~m}$ & $19 \mathrm{~m}$ \\
$\lambda_{\mathrm{s}, 50 \mathrm{~Hz}}$ & $4 \mathrm{~m}$ & $11 \mathrm{~m}$ \\
\hline
\end{tabular}

The elastodynamic problem is solved using the finite element method with two-dimensional four-node elements in plane strain. For the mesh, an element size of $0.25 \mathrm{~m}$ is used, corresponding to ten elements per shear wavelength $\lambda_{\mathrm{s}}$ at a frequency of $80 \mathrm{~Hz}$, which is the upper limit considered in this paper. The discretization results in a displacement vector $\hat{\mathbf{u}}$ collecting $N_{\text {dof }}$ degrees of freedom, determined by the following system of equations:

$$
\hat{\mathbf{K}} \hat{\mathbf{u}}=\hat{\mathbf{p}}
$$

In this expression, $\hat{\mathbf{u}}$ is the displacement vector, $\hat{\mathbf{p}}$ is the load vector, and $\hat{\mathbf{K}}$ is the dynamic stiffness matrix. The dynamic stiffness matrix is computed as:

$$
\hat{\mathbf{K}}=-\omega^{2} \mathbf{M}+\mathbf{K}
$$

with $\omega$ the considered frequency $[\mathrm{rad} / \mathrm{s}], \mathbf{M}$ the mass matrix, and $\mathbf{K}$ the stiffness matrix, which are assembled from the element mass and stiffness matrices. The element mass matrix $\mathbf{M}_{e}$ and the element stiffness matrix $\mathbf{K}_{e}$ are calculated from the matrix $\mathbf{N}$ containing the shape functions, the matrix $\mathbf{B}$ containing the derivatives of the shape functions, and the constitutive matrix $\mathbf{D}$. In the case of plane strain, the following expressions are obtained:

$$
\mathbf{M}_{e}=\int_{\Omega} \mathbf{N}^{T} \rho \mathbf{N} d \Omega=\rho \mathbf{M}_{0, e}
$$




$$
\mathbf{K}_{e}=\int_{\Omega} \mathbf{B}^{T} \mathbf{D B} d \Omega=\rho C_{\mathrm{p}}^{2} \mathbf{K}_{\mathrm{p}, e}+\rho C_{\mathrm{s}}^{2} \mathbf{K}_{\mathrm{s}, e}
$$

The resulting $8 \times 8$ matrices $\mathbf{M}_{0, e}, \mathbf{K}_{\mathrm{p}, e}$, and $\mathbf{K}_{\mathrm{s}, e}$ only depend on the element sizes. Note that $\rho C_{\mathrm{p}}^{2}=\lambda+2 \mu$ (with $\lambda$ and $\mu$ the Lamé coefficients) is the constrained modulus and $\rho C_{\mathrm{s}}^{2}=\mu$ is the shear modulus.

A hysteretic damping model is applied. According to the correspondence principle, this can be modeled in the frequency domain by the introduction of complex material properties, denoted by a prime, with damping ratios $\beta_{\mathrm{p}}$ and $\beta_{\mathrm{s}}$ :

$$
\begin{aligned}
& \left(\rho C_{\mathrm{p}}^{2}\right)^{\prime}=\rho C_{\mathrm{p}}^{2}\left(1+2 \mathrm{i} \beta_{\mathrm{p}}\right) \\
& \left(\rho C_{\mathrm{s}}^{2}\right)^{\prime}=\rho C_{\mathrm{s}}^{2}\left(1+2 \mathrm{i} \beta_{\mathrm{s}}\right)
\end{aligned}
$$

with $\mathrm{i}$ the imaginary unit. However, no damping was included to obtain the results in this paper, and therefore the damping ratios are $\beta_{\mathrm{p}}=\beta_{\mathrm{s}}=0$.

At the boundaries of the finite element mesh, appropriate radiation boundary conditions have to prevent spurious wave reflections. Several solutions exist, such as absorbing boundary conditions that minimize the reflectance [17], Perfectly Matched Layers (PML) [18], the boundary element method [19], etc. In this article, the PMLs of Harari and Albocher [20, 21] are applied, where a complex coordinate stretch is used to calculate the element mass and the element stiffness matrices:

$$
\begin{aligned}
& \tilde{x}=\int_{0}^{x} \psi_{x}(\xi) \mathrm{d} \xi \\
& \tilde{y}=\int_{0}^{y} \psi_{y}(\eta) \mathrm{d} \eta
\end{aligned}
$$

where $\tilde{x}$ and $\tilde{y}$ are the stretched coordinates of coordinates $x$ and $y$. The complex-valued stretch function is defined as:

$$
\psi_{j}=1+\mathrm{i} s_{j}
$$

with $s_{j}$ the attenuation function in direction $j(x$ or $y$ ). The stretch functions depend on the attenuation direction in the PML. In the region indicated by number 1 in figure 1 , the attenuation will only take place in the horizontal direction, and the stretch functions are $\psi_{x}=1+\mathrm{i} s_{x}(x)$ and $\psi_{y}=1$. A quadratic function of the coordinate $x$ is chosen as attenuation function:

$$
s_{x}(x)=s_{\max }\left(\frac{x}{L}\right)^{2}, \quad 0<x<L
$$

where a maximum value $s_{\max }$ of 13.2 is chosen at $25 \mathrm{~Hz}$ and a value of 6.6 at $50 \mathrm{~Hz}$. Similarly, in region 2 the stretch functions are $\psi_{x}=1$ and $\psi_{y}=1+\mathrm{i} s_{y}(y)$ and in region $3 \psi_{x}=1+\mathrm{i} s_{x}(x)$ and $\psi_{y}=1+\mathrm{i} s_{y}(y)$.

In topology optimization, the topology is parametrized by densities attributed to the finite elements in the design domain. In order to achieve a design with sufficient detail, the finite element mesh of the design domain is finer than the one of the surrounding domain, following the method proposed by Kristensen [21]. The horizontal and vertical displacement of the intermediate nodes are coupled to the nodes of the surrounding domain, resulting in constraint equations which can be written in matrix form:

$$
\mathrm{Q} \hat{\mathbf{u}}=\mathbf{0}
$$

where the matrix $\mathbf{Q}$ collects the coefficients.

Different methods exist to enforce these constraints, e.g. using a transformation matrix, adding penalty functions, using Lagrange multipliers, etc. [22, chapter 13]. Here, Lagrange multipliers are used, leading to the following system of equations:

$$
\left[\begin{array}{cc}
\hat{\mathbf{K}} & \mathbf{Q}^{T} \\
\mathbf{Q} & \mathbf{0}
\end{array}\right]\left\{\begin{array}{l}
\hat{\mathbf{u}} \\
\chi
\end{array}\right\}=\left\{\begin{array}{l}
\hat{\mathbf{p}} \\
\mathbf{0}
\end{array}\right\}
$$

where $\chi$ are the Lagrange multipliers. 


\subsection{Introduction to topology optimization}

Topology optimization searches the optimal distribution of material in a design domain taking into account a set of relevant constraints [1]. The material distribution is parameterized using element densities $\bar{\rho}_{e}$ for each element $e$ in the design domain. These element densities can take values in the interval $[0,1]$, where $\bar{\rho}_{e}=0$ indicates that element $e$ has the properties of the original material (material 1), while $\bar{\rho}_{e}=1$ corresponds to the properties of the stiffened material (material 2). By allowing the densities to vary continuously between 0 and 1 , the optimization problem can be solved with a gradient based optimization algorithm. The method of moving asymptotes (MMA) [23] is used.

The (physical) element densities $\bar{\rho}_{e}$ are obtained from the design variables $\rho_{e}$ of the optimization problem by applying a projection filter [24]:

$$
\bar{\rho}_{e}=\frac{\tanh (\beta \eta)+\tanh \left(\beta\left(\tilde{\rho}_{e}-\eta\right)\right)}{\tanh (\beta \eta)+\tanh (\beta(1-\eta))}
$$

where $\beta$ is a sharpness parameter, controlling the smoothness of the projection, $\eta$ is the projection threshold, and the densities $\tilde{\rho}_{e}$ are obtained from:

$$
\tilde{\rho}_{e}=\frac{\sum_{i=1}^{N} w_{e i} v_{i} \rho_{i}}{\sum_{i=1}^{N} w_{e i} v_{i}}
$$

where $v_{i}$ is the volume of element $i$ and the weight $w_{e i}=\max \left(R-r_{e i}\right)$ depends on the filter radius $R$ and the center-to-center distance $r_{e i}$ between the elements $e$ and $i$. In the present work, the filter radius is taken to be $R=2.5$ elements, the projection threshold value is set to $\eta=0.5$ and the sharpness parameter $\beta$ has an initial value equal to 1 and is doubled every 50 iterations until the value of 32 is reached.

A Solid Isotropic Material with Penalization (SIMP) interpolation $[25,26]$ is used to interpolate the material properties $\alpha$ :

$$
\alpha=\alpha_{1}+\bar{\rho}_{e}^{p}\left(\alpha_{2}-\alpha_{1}\right)
$$

where $\alpha_{1}$ and $\alpha_{2}$ are the properties of the original and stiffened material, respectively. The penalization factor $p \geq 1$ avoids so-called gray designs with intermediate densities. A value $p=1$ is used for the mass density $\rho$ while $p=3$ is used for the constrained modulus $\left(\rho C_{\mathrm{p}}^{2}\right)^{\prime}$ and the shear modulus $\left(\rho C_{\mathrm{s}}^{2}\right)^{\prime}$.

\subsection{The optimization problem}

Consider again the topology optimization problem shown in figure 1 . The aim is to maximize the insertion loss (IL), that quantifies the effectiveness of the wave barrier as:

$$
\mathrm{IL}=10 \log \left(\frac{\hat{\mathbf{u}}^{\mathrm{orig} H} \mathbf{L} \hat{\mathbf{u}}^{\text {orig }}}{\hat{\mathbf{u}}^{H} \mathbf{L} \hat{\mathbf{u}}}\right)
$$

with $(\cdot)^{H}$ the Hermitian (or conjugate) transpose, $\hat{\mathbf{u}}^{\text {orig }}$ the displacement vector of the original field (the homogeneous halfspace) and $\hat{\mathbf{u}}$ the displacement vector of the actual field. $\mathbf{L}$ is a sparse selection matrix, containing ones at the diagonal elements corresponding to the degrees of freedom selected for assessing the effectiveness of the wave barrier. The problem is reformulated as a minimization problem, resulting in an objective function $\Phi=-\mathrm{IL}$, and is subjected to a volume constraint:

$$
\begin{array}{ll}
\min _{\rho_{e}} & 10 \log \left(\frac{\hat{\mathbf{u}}^{H} \mathbf{L} \hat{\mathbf{u}}}{\hat{\mathbf{u}}^{\text {orig } H} \mathbf{L} \hat{\mathbf{u}}^{\text {orig }}}\right) \\
\text { s.t. } & \sum_{e=1}^{N} v_{e} \bar{\rho}_{e} \leq V^{*}, e=1 \ldots N \\
& 0 \leq \rho_{e} \leq 1, e=1 \ldots N \\
\text { with } & {\left[\begin{array}{cc}
\hat{\mathbf{K}} & \mathbf{Q}^{T} \\
\mathbf{Q} & \mathbf{0}
\end{array}\right]\left\{\begin{array}{l}
\hat{\mathbf{u}} \\
\chi
\end{array}\right\}=\left\{\begin{array}{l}
\hat{\mathbf{p}} \\
\mathbf{0}
\end{array}\right\}}
\end{array}
$$


where $N$ is the number of elements in the design domain. The upper limit for the volume $V^{*}$ is set to $20 \%$ of the total volume. Note that this problem is not convex, and multiple local minima may be present.

As a gradient-based approach is used, the derivatives of the objective function are required. The adjoint method is an efficient method to compute the gradient in the case of a large number of design variables and is therefore applied. Appendix A addresses the derivation of the sensitivities, which leads to:

$$
\frac{\partial \Phi}{\partial \bar{\rho}_{e}}=2 \operatorname{Re}\left\{\lambda^{T} \frac{\partial \hat{\mathbf{K}}}{\partial \bar{\rho}_{e}} \hat{\mathbf{u}}\right\}
$$

with $\boldsymbol{\lambda}$ computed from the adjoint equation:

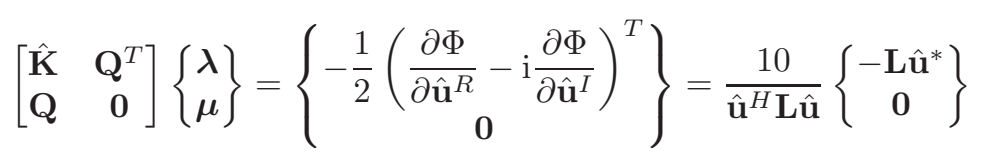

The sensitivities in Eq. (18) are (in the case of plane strain) straightforwardly computed from the elaborated element mass and stiffness matrix in Eq. (3) and (4), respectively:

$$
\begin{aligned}
\frac{\partial \hat{\mathbf{K}}_{e}}{\partial \bar{\rho}_{e}} & =-\omega^{2} \frac{\partial(\rho)}{\partial \bar{\rho}_{e}} \mathbf{M}_{0, e}+\frac{\partial\left(\rho C_{\mathrm{p}}^{2}\right)}{\partial \bar{\rho}_{e}} \mathbf{K}_{\mathrm{p}, e}+\frac{\partial\left(\rho C_{\mathrm{s}}^{2}\right)}{\partial \bar{\rho}_{e}} \mathbf{K}_{\mathrm{s}, e} \\
& =-\omega^{2}\left(\rho_{2}-\rho_{1}\right) \mathbf{M}_{0, e}+p \bar{\rho}_{e}^{p-1}\left[\left(\rho_{2} C_{\mathrm{p} 2}^{2}-\rho_{1} C_{\mathrm{p} 1}^{2}\right) \mathbf{K}_{\mathrm{p}, e}+\left(\rho_{2} C_{\mathrm{s} 2}^{2}-\rho_{1} C_{\mathrm{s} 1}^{2}\right) \mathbf{K}_{\mathrm{s}, e}\right]
\end{aligned}
$$

\section{Optimization of wave barriers for harmonic waves}

In this section, harmonic vibration sources are considered. As initial design, all element densities are assigned a value $\rho_{e}=0.2$. Two cases are considered. First, only the vertical component of the displacement at the output point is considered in the calculation of the insertion loss (and therefore the objective function). Second, the norm of the displacement vector is considered.

When only the vertical displacement in the output point is considered, the insertion loss becomes:

$$
\mathrm{IL}_{y}=10 \log \left(\frac{\hat{\mathbf{u}}^{\text {orig } H} \mathbf{L} \hat{\mathbf{u}}^{\text {orig }}}{\hat{\mathbf{u}}^{H} \mathbf{L} \hat{\mathbf{u}}}\right)=10 \log \left(\frac{\left|\hat{u}_{\text {out }, y}^{\text {orig }}\right|^{2}}{\left|\hat{u}_{\text {out }, y}\right|^{2}}\right)=20 \log \left(\left|\frac{\hat{u}_{\text {out }, y}^{\text {orig }}}{\hat{u}_{\text {out }, y}}\right|\right)
$$

The insertion loss of the optimized design and a reference rectangular design with dimensions $1 \mathrm{~m} \times 8 \mathrm{~m}$ are compared in the following. The reference design, the design optimized for harmonic excitation at $25 \mathrm{~Hz}$ and the corresponding insertion loss $\mathrm{IL}_{y}$ are shown in figure 2.

In the neighborhood of the targeted frequency of $25 \mathrm{~Hz}$, the insertion loss obtained for the optimized design is positive and significantly larger than for the reference design. At higher frequencies, from around $50 \mathrm{~Hz}$, the optimized design performs worse than the reference design and at frequencies above $60 \mathrm{~Hz}$, the insertion loss of the optimized design is close to zero. In this frequency range, the penetration depth of the Rayleigh waves is too small, so that they are not affected by the stiffened material introduced at depth. At frequencies above $80 \mathrm{~Hz}$, the transmission of the Rayleigh waves is affected by the zone at the surface, providing again a positive value for the insertion loss, but smaller than for the reference design.

The optimized design is symmetric due to the symmetric nature of the problem. Both the load $\hat{\mathbf{p}}$ and the adjoint load $\frac{-10 \mathbf{L} \hat{\mathbf{u}}^{*}}{\hat{\mathbf{u}}^{H} \mathbf{L} \hat{\mathbf{u}}}$ have only a single entry, at degrees of freedom that are positioned symmetrically with respect to the design domain. This makes the sensitivities in Eq. (18) symmetrical, resulting in a symmetric design.

In order to verify the reduction of the horizontal response in the output point obtained by this optimized design, the insertion losses $\mathrm{IL}_{x}$ and $\mathrm{IL}_{x y}$ are defined as:

$$
\mathrm{IL}_{x}=10 \log \left(\frac{\left|\hat{u}_{\text {out }, x}^{\text {orig }}\right|^{2}}{\left|\hat{u}_{\text {out }, x}\right|^{2}}\right)=20 \log \left(\left|\frac{\hat{u}_{\text {out }, x}^{\text {orig }}}{\hat{u}_{\text {out }, x}}\right|\right)
$$




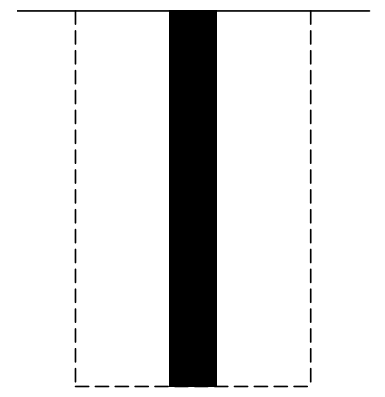

(a)

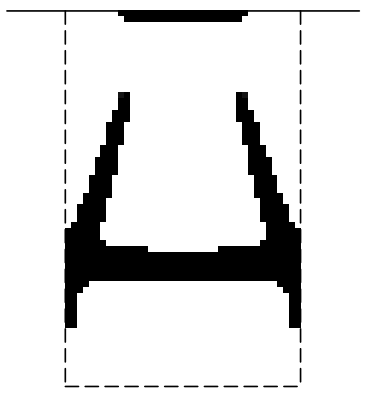

(b)

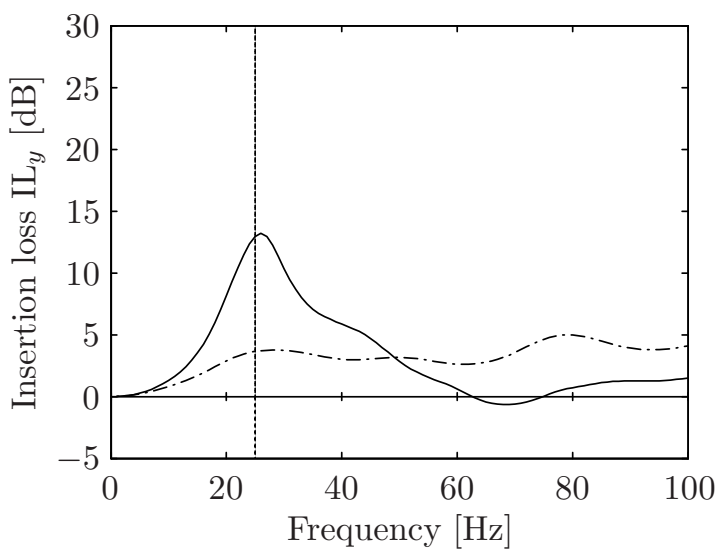

(c)

Figure 2: (a) Reference design, (b) optimized design maximizing $\mathrm{IL}_{y}$ at $25 \mathrm{~Hz}$, and (c) resulting insertion loss $\mathrm{IL}_{y}$ (Eq. (21)) as a function of the frequency for the reference design (dash-dotted line) and the optimized design (solid line).

$$
\mathrm{IL}_{x y}=10 \log \left(\frac{\left|\hat{u}_{\text {out }, x}^{\text {orig }}\right|^{2}+\left|\hat{u}_{\text {out }, y}^{\text {orig }}\right|^{2}}{\left|\hat{u}_{\text {out }, x}\right|^{2}+\left|\hat{u}_{\text {out }, y}\right|^{2}}\right)=20 \log \left(\frac{\sqrt{\left|\hat{u}_{\text {out }, x}^{\text {orig }}\right|^{2}+\left|\hat{u}_{\text {out }, y}^{\text {orig }}\right|^{2}}}{\sqrt{\left|\hat{u}_{\text {out }, x}\right|^{2}+\left|\hat{u}_{\text {out }, y}\right|^{2}}}\right)
$$

The insertion losses $\mathrm{IL}_{x}$ and $\mathrm{IL}_{x y}$ for the optimized design in figure $2 \mathrm{~b}$ are shown in figure 3 . The design optimized for the vertical response at $25 \mathrm{~Hz}$ leads to a much higher horizontal response at $25 \mathrm{~Hz}$ than the reference design and the response is even higher than for the original homogeneous halfspace. As a consequence, the insertion loss of the vector sum $\mathrm{IL}_{x y}$ of the optimized design is hardly higher than for the reference design.

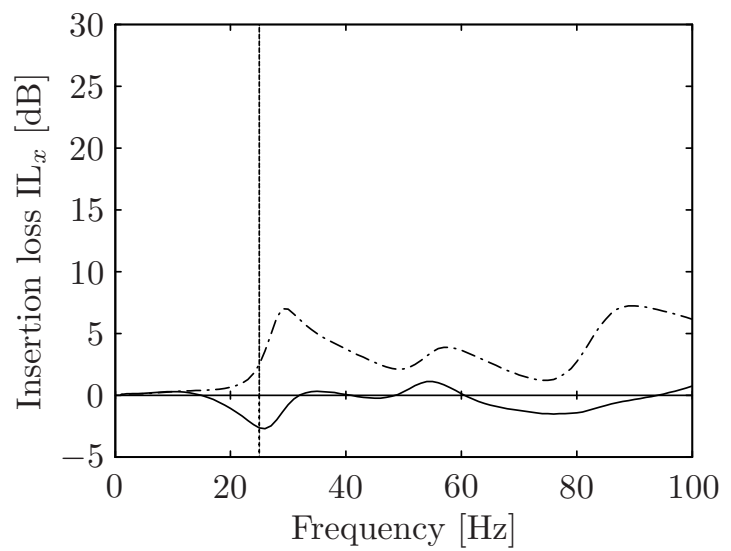

(a)

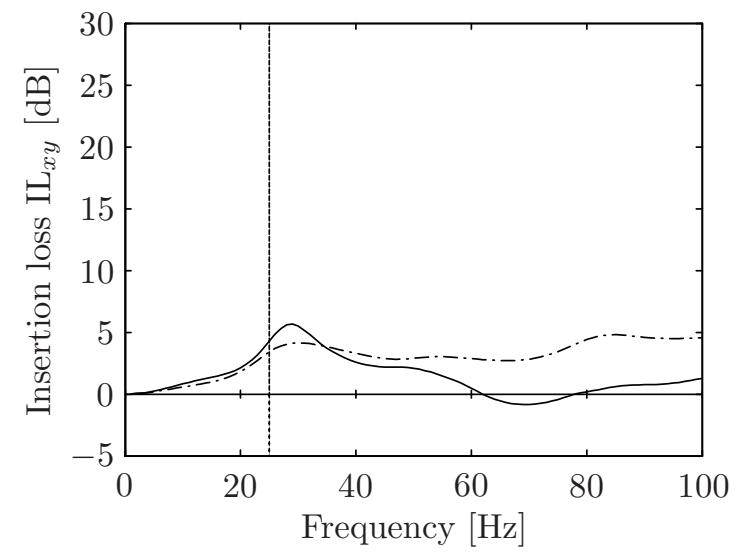

(b)

Figure 3: The insertion loss (a) $\mathrm{IL}_{x}$ (Eq. (22)) and (b) $\mathrm{IL}_{x y}$ (Eq. (23)) as a function of the frequency for the reference design in figure $2 \mathrm{a}$ (dash-dotted line) and the optimized design in figure $2 \mathrm{~b}$ (solid line).

Maximizing the insertion loss of the vector sum in Eq. (23) for a harmonic point source at $25 \mathrm{~Hz}$ results in the design and insertion loss $\mathrm{IL}_{x y}$ in figure 4. Since the horizontal displacement is introduced in the selection matrix $\mathbf{L}$, the optimized design is no longer symmetric. Comparing figures $3 \mathrm{~b}$ and $4 \mathrm{~b}$ shows that the resulting insertion loss $\mathrm{IL}_{x y}$ is considerably higher. At $25 \mathrm{~Hz}$, the optimized design leads to a reduction 
of $7.2 \mathrm{~dB}$ compared to the original situation which is $3.8 \mathrm{~dB}$ higher than the insertion loss of the reference design in figure $2 \mathrm{a}$.

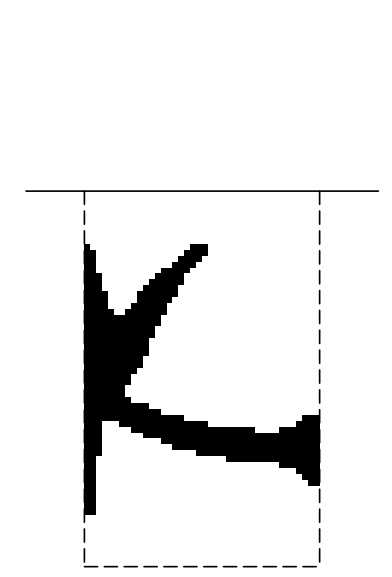

(a)

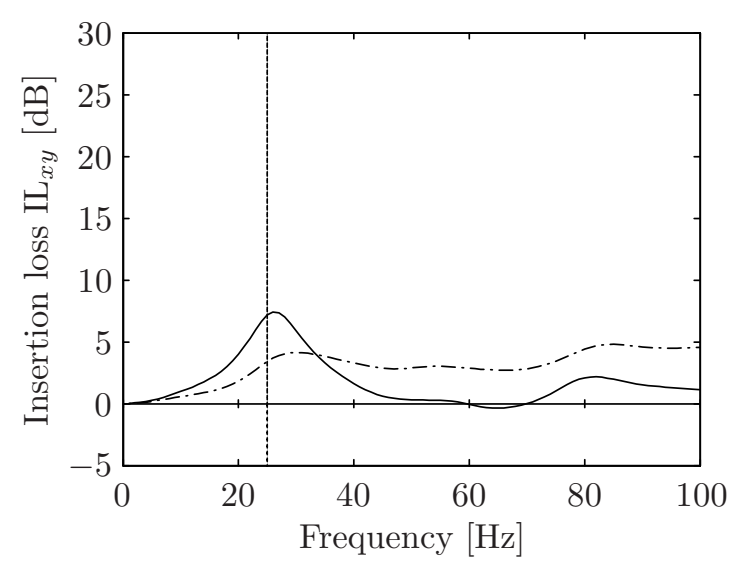

(b)

Figure 4: (a) Optimized design maximizing $\mathrm{IL}_{x y}$ at $25 \mathrm{~Hz}$, and (b) resulting insertion loss $\mathrm{IL}_{x y}$ (Eq. (23)) as a function of the frequency for the reference design in figure $2 \mathrm{a}$ (dash-dotted line) and the optimized design (solid line).

The optimized design in figure 4 a consists of three main features: (1) the vertical part at the left hand side of the design domain, (2) the inclined part going upward from the center of this vertical part, and (3) the curved part from the center of the vertical part to the right hand side of the design domain. In order to verify the role of these features, figure 5 shows the real part of the displacement field and the insertion loss at $25 \mathrm{~Hz}$ in the surrounding domain for the homogeneous halfspace, for the reference design, for part (1) of the optimized design, and for the entire design. The vertical part (1) works mainly as a traditional wall barrier that reflects the incoming waves. Since the design domain comprising the optimal wave barriers is located at the surface of the halfspace, the incoming waves are mainly surface or Rayleigh waves. The Rayleigh waves in a halfspace consisting of solely material 1 (with the properties given in table 1) have a wave speed $C_{\mathrm{R}}$ equal to $186 \mathrm{~m} / \mathrm{s}$, or a wavelength $\lambda_{\mathrm{R}}$ equal to $7.5 \mathrm{~m}$ at $25 \mathrm{~Hz}$. The depth of the vertical part extends more or less from $0.2 \lambda_{\mathrm{R}}$ to $0.9 \lambda_{\mathrm{R}}$, in line with the rule of thumb stating that open trenches start to be effective in cutting off the propagation of Rayleigh waves if the depth of the trench is at least half the Rayleigh wavelength [27]. By adding the two other parts (2) and (3), the waves are directed in the extension of their branches. The inclined part (2) directs the waves primarily to the surface, where they are reflected downwards in the domain together with the waves directed by the curved part (3), reducing the response at the surface.

The optimized design and its performance change significantly when the wavelength becomes smaller. Consider for example excitation at $50 \mathrm{~Hz}$. The optimized design and the resulting insertion loss $\mathrm{IL}_{x y}$ are shown in figure 6 . The peak in the insertion loss at $50 \mathrm{~Hz}$ reaches a very high value of $59.3 \mathrm{~dB}$, and is much higher than for the reference design in figure $2 \mathrm{a}$, where the insertion loss reaches a value of $2.9 \mathrm{~dB}$ at $50 \mathrm{~Hz}$.

In the optimized design of figure $6 \mathrm{a}$, material 2 is introduced in three main areas: (1) the top part, partially horizontal and partially inclined, (2) the middle part situated approximately at a depth of $\lambda_{\mathrm{R}}$ consisting of two small inclusions, and (3) the curved bottom part at a depth of $2 \lambda_{\mathrm{R}}$. Figure 7 shows the real part of the displacement field and the insertion loss at $50 \mathrm{~Hz}$ in the surrounding domain for the homogeneous halfspace, for the reference design, for the left half of part (1) of the optimized design, for part (1) of the optimized design, and for the entire design. The left half of the top part (1) splits the incoming Rayleigh waves with a wavelength equal to $3.7 \mathrm{~m}$ at $50 \mathrm{~Hz}$ in two waves, one propagating through the stiffened material and one redirected downwards. The depth of this part is equal to $0.2 \lambda_{\mathrm{R}}$ and the resulting insertion loss of this part of the design is already close to the insertion loss of the reference design in figure 2a. The right half acts again as a waveguide and directs the waves downwards, under the surface, outperforming the 


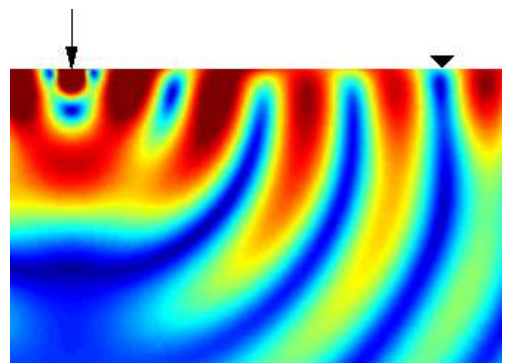

(a)

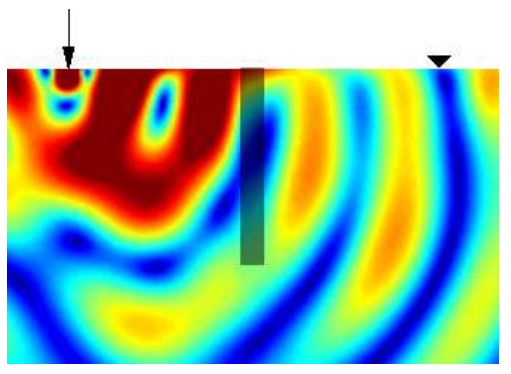

(b)

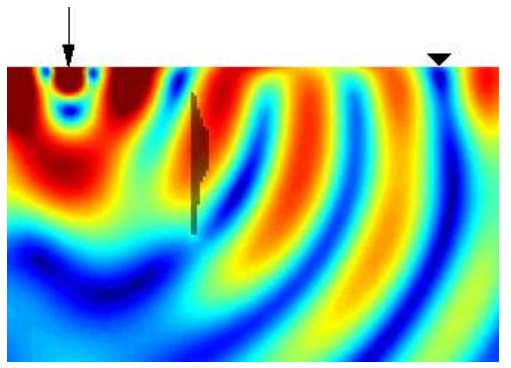

(c)

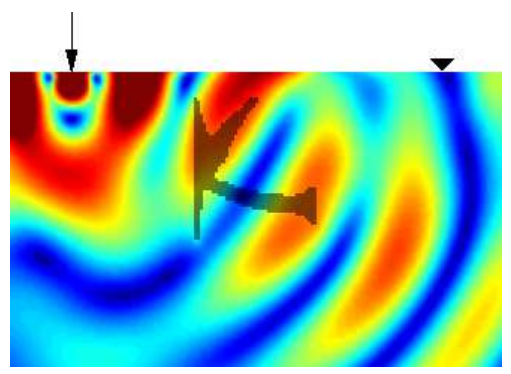

(d)
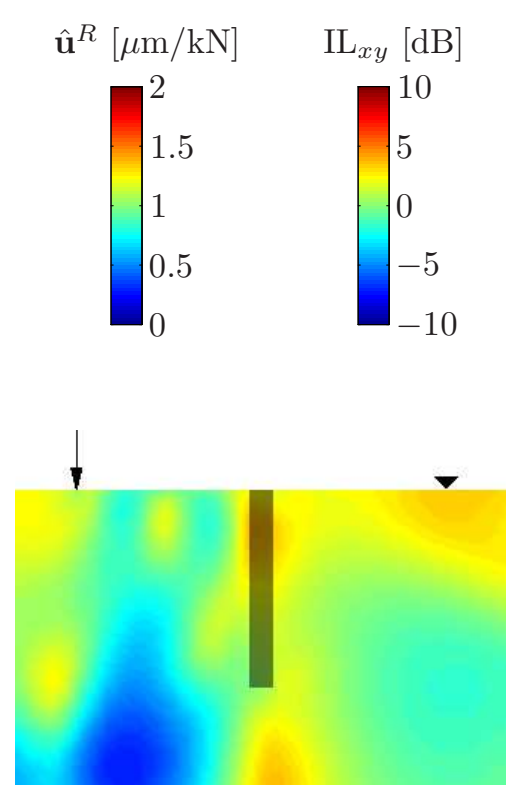

(e)

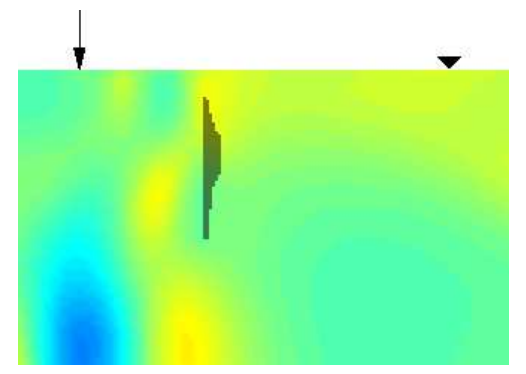

(f)

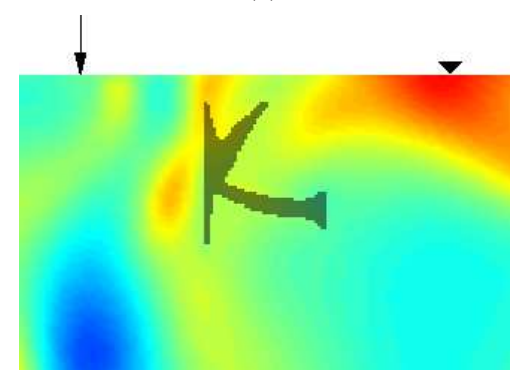

(g)

Figure 5: Real part of the displacement field $\hat{\mathbf{u}}^{R}$ at $25 \mathrm{~Hz}$ (a) for the original domain and after the introduction of (b) the reference design in figure $2 \mathrm{a}$, (c) part (1) of the optimized design, and (d) the entire optimized design maximizing $\mathrm{IL}_{x y}$ at $25 \mathrm{~Hz}$ (figure $4 \mathrm{a}$ ). Insertion loss $\mathrm{IL}_{x y}$ at $25 \mathrm{~Hz}$ after the introduction of (e) the reference design in figure $2 \mathrm{a}$, (f) part (1) of the optimized design, and $(\mathrm{g})$ the entire optimized design maximizing $\mathrm{IL}_{x y}$ at $25 \mathrm{~Hz}$ (figure $4 \mathrm{a}$ ).

reference design already by $5.6 \mathrm{~dB}$ (compare figures $7 \mathrm{f}$ and $7 \mathrm{~h}$ ). The waves that are redirected downwards by the top part, are guided and reflected by the mid part (2) and the bottom part (3) and finally interfere with the waves transmitted by the top part, minimizing the displacement at the surface. This destructive interference is very sensitive to geometric imperfections, as will be shown in section 5 . 


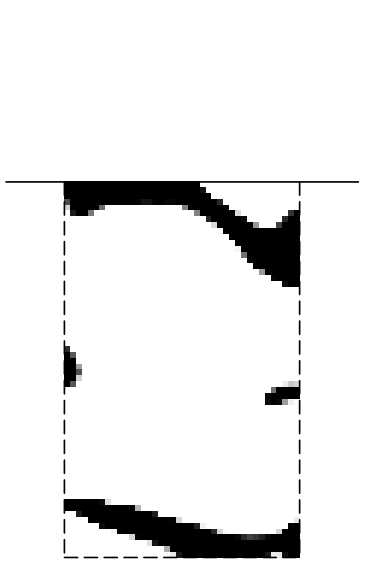

(a)

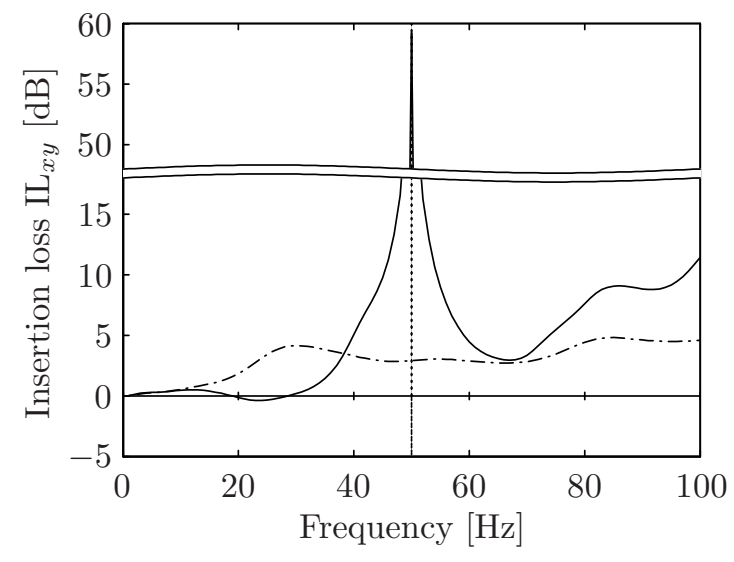

(b)

Figure 6: (a) Optimized design maximizing $\mathrm{IL}_{x y}$ at $50 \mathrm{~Hz}$, and (b) resulting insertion loss $\mathrm{IL}_{x y}($ Eq. (23)) as a function of the frequency for the reference design in figure $2 \mathrm{a}$ (dash-dotted line) and the optimized design (solid line).

\section{Optimization of wave barriers for a target frequency range}

In the previous section, the optimization was performed for harmonic excitation at a single fixed frequency. In this section, a frequency range $\left[f_{1}, f_{2}\right]$ is considered. A distinction is made between (1) harmonic excitation at a frequency in a given range and (2) broadband excitation. For harmonic sources at a frequency in a given range, a good performance is needed for the entire frequency range, and the minimal insertion loss over the frequency range is maximized. For broadband sources, the frequency averaged insertion loss is maximized.

If the objective is to maximize the minimal insertion loss, the optimization problem becomes:

$$
\begin{array}{ll}
\min _{\rho_{e}} & \max _{\substack{f_{n} \\
n=1 \ldots N_{\mathrm{f}}}} 10 \log \left(\frac{\left|\hat{u}^{\text {out }, x}\left(f_{n}\right)\right|^{2}+\left|\hat{u}^{\text {out }, y}\left(f_{n}\right)\right|^{2}}{\left|\hat{u}_{\text {orig }}^{\text {out } x}\left(f_{n}\right)\right|^{2}+\left|\hat{u}_{\text {orig }}^{\text {out } y}\left(f_{n}\right)\right|^{2}}\right) \\
\text { s.t. } & \sum^{N} v_{e} \bar{\rho}_{e} \leq V^{*}, e=1 \ldots N \\
& 0 \leq \rho_{e} \leq 1, e=1 \ldots N \\
\text { with } \quad\left[\begin{array}{cc}
\hat{\mathbf{K}} & \mathbf{Q}^{T} \\
\mathbf{Q} & \mathbf{0}
\end{array}\right]\left\{\begin{array}{l}
\hat{\mathbf{u}} \\
\boldsymbol{\chi}
\end{array}\right\}=\left\{\begin{array}{l}
\hat{\mathbf{p}} \\
\mathbf{0}
\end{array}\right\}
\end{array}
$$

The frequency range is discretized using $N_{\mathrm{f}}=10$ equidistant frequencies. Increasing the number of frequencies $N_{\mathrm{f}}$ hardly changes the optimal design geometry, indicating that only taking 10 frequencies into account is sufficient. This problem is not differentiable and is therefore reformulated using a bound formulation [28]. Figure 8 shows the design optimized for the frequency range from 20 to $80 \mathrm{~Hz}$, corresponding to Rayleigh wavelengths between 2.3 and $9.3 \mathrm{~m}$, and the resulting insertion loss. The insertion loss is higher than for the reference design in the entire frequency range considered. The optimized design contains a part (1) that maximizes the insertion loss in the lower frequency range (bottom left, compare with figure 4a) and a part (2) for the higher frequency range (top right, compare with figure 6a).

If the objective is to maximize the frequency averaged insertion loss $\overline{\mathrm{IL}_{x y}}$ (in the case of broadband sources), the parameter to be optimized becomes:

$$
\overline{\mathrm{IL}_{x y}}=\frac{1}{f_{2}-f_{1}} \int_{f_{1}}^{f_{2}} \mathrm{IL}_{x y}(f) \mathrm{d} f=\frac{10}{f_{2}-f_{1}} \int_{f_{1}}^{f_{2}} \log \left(\frac{\left|\hat{u}_{\text {orig }}^{\text {out }, x}(f)\right|^{2}+\left|\hat{u}_{\text {orig }}^{\text {out }, y}(f)\right|^{2}}{\left|\hat{u}^{\text {out }, x}(f)\right|^{2}+\left|\hat{u}^{\text {out }, y}(f)\right|^{2}}\right) \mathrm{d} f
$$




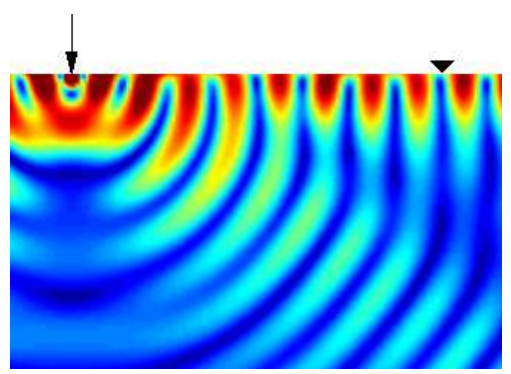

(a)

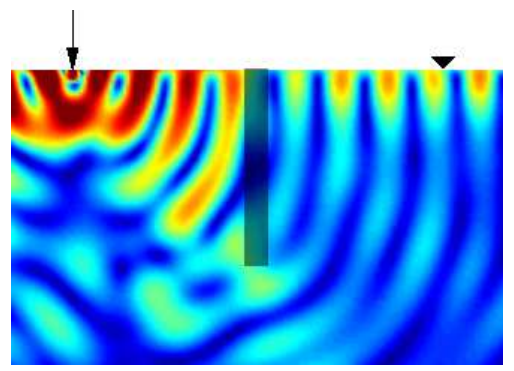

(b)

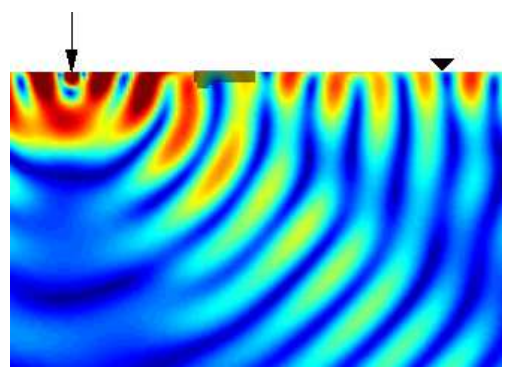

(c)

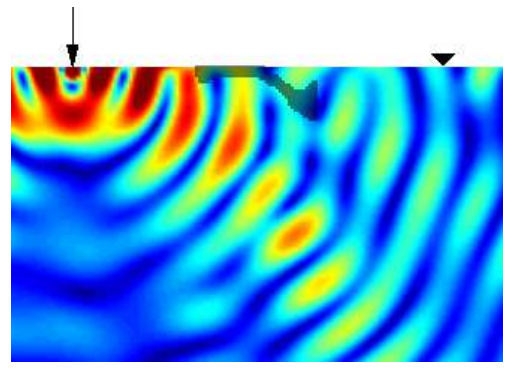

(d)

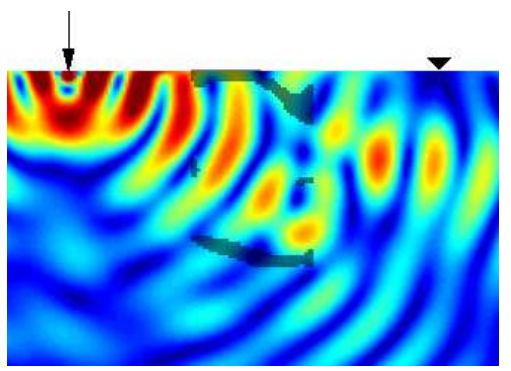

(e)
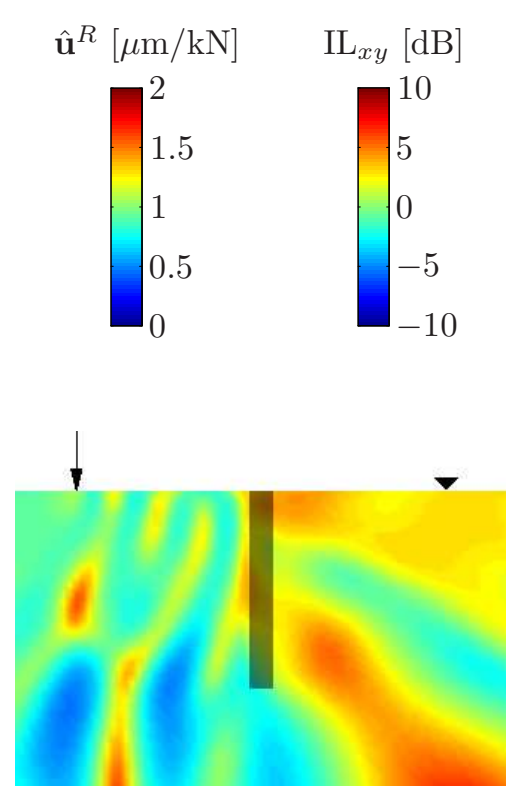

(f)

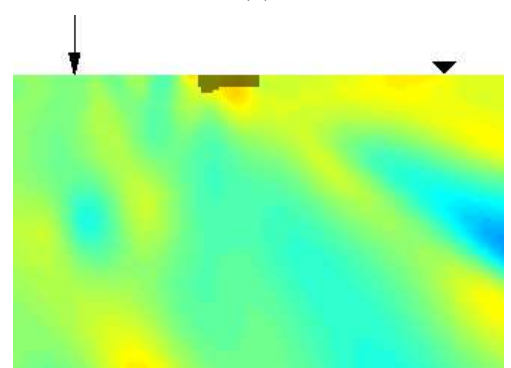

(g)

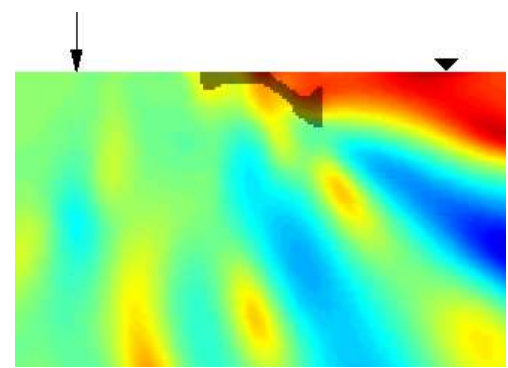

(h)

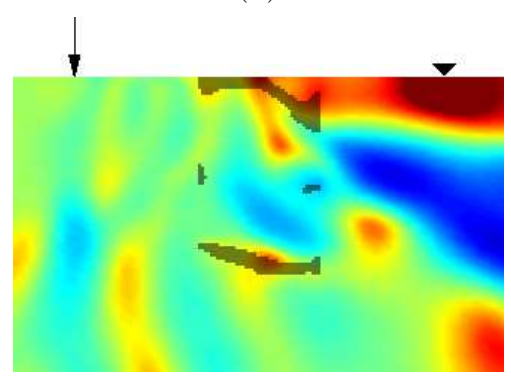

(i)

Figure 7: Real part of the displacement field $\hat{\mathbf{u}}^{R}$ at $50 \mathrm{~Hz}$ (a) for the original domain and after the introduction of (b) the reference design in figure 2a, (c) the left half of part (1) of the optimized design, (d) part (1) of the optimized design, and (e) the entire optimized design maximizing $\mathrm{IL}_{x y}$ at $50 \mathrm{~Hz}$ (figure 6a). Insertion loss $\mathrm{IL}_{x y}$ at $50 \mathrm{~Hz}$ after the introduction of (f) the reference design in figure $2 \mathrm{a},(\mathrm{g})$ the left half of part (1) of the optimized design, (h) part (1) of the optimized design, and (i) the entire optimized design maximizing $\mathrm{IL}_{x y}$ at $50 \mathrm{~Hz}$ (figure 6a). 


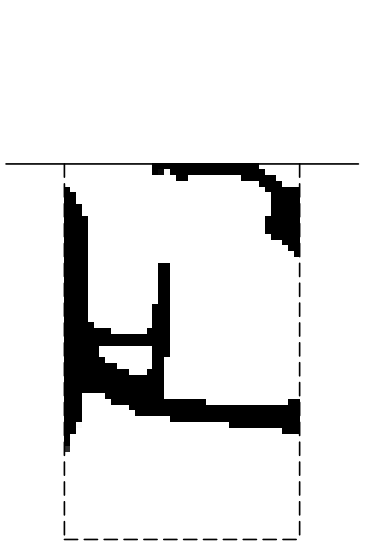

(a)

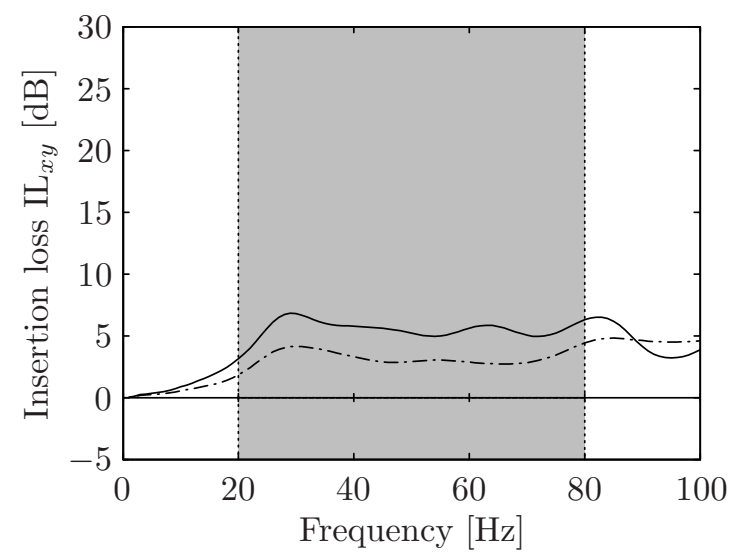

(b)

Figure 8: (a) Optimized design maximizing $\min _{f_{n}} \mathrm{IL}_{x y}\left(f_{n}\right)$ for the frequency range $20-80 \mathrm{~Hz}$ (discretized with 10 frequencies), and (b) resulting insertion loss $\mathrm{IL}_{x y}$ (Eq. (23)) as a function of the frequency for the reference design in figure 2a (dash-dotted line) and the optimized design (solid line).

The optimization problem minimizes the objective function $\Phi^{\prime}=-\overline{\mathrm{IL}_{x y}}$, where the integral in Eq. (25) is approximated by a discretization of the frequency range by $N_{\mathrm{f}}$ equidistant frequencies.

$$
\begin{array}{ll}
\min _{\rho_{e}} & \frac{10}{N_{\mathrm{f}}} \sum_{n=1}^{N_{\mathrm{f}}} \log \left(\frac{\left|\hat{u}^{\text {out }, x}\left(f_{n}\right)\right|^{2}+\left|\hat{u}^{\text {out }, y}\left(f_{n}\right)\right|^{2}}{\left|\hat{u}_{\text {orig }}^{\text {out }, x}\left(f_{n}\right)\right|^{2}+\left|\hat{u}_{\text {orig }}^{\text {out }, y}\left(f_{n}\right)\right|^{2}}\right) \\
\text { s.t. } & \sum_{e=1}^{N} v_{e} \bar{\rho}_{e} \leq V^{*}, e=1 \ldots N \\
& 0 \leq \rho_{e} \leq 1, e=1 \ldots N \\
\text { with } \quad & {\left[\begin{array}{cc}
\hat{\mathbf{K}} & \mathbf{Q}^{T} \\
\mathbf{Q} & \mathbf{0}
\end{array}\right]\left\{\begin{array}{l}
\hat{\mathbf{u}} \\
\chi
\end{array}\right\}=\left\{\begin{array}{l}
\hat{\mathbf{p}} \\
\mathbf{0}
\end{array}\right\}}
\end{array}
$$

Figure 9 shows the design optimized for the frequency range from 20 to $80 \mathrm{~Hz}$, using $N_{\mathrm{f}}=10$ frequencies in Eq. (26), and the resulting insertion loss $\mathrm{IL}_{x y}$. The optimization leads to designs which mainly reduce transmission at higher frequencies, as it is easier to obtain high insertion loss values in this frequency range, as observed in section 3. Figure 10 shows the real part of the displacement field for frequencies of $30 \mathrm{~Hz}$ $\left(\lambda_{\mathrm{R}}=6.2 \mathrm{~m}\right), 50 \mathrm{~Hz}\left(\lambda_{\mathrm{R}}=3.7 \mathrm{~m}\right)$, and $70 \mathrm{~Hz}\left(\lambda_{\mathrm{R}}=2.7 \mathrm{~m}\right)$ in the surrounding domain for the homogeneous halfspace, the reference design and the design optimized for the average insertion loss in the frequency range $20-80 \mathrm{~Hz}$ (figure 9a). For the highest frequencies of the target range, almost all the energy is reflected by the structure, resulting in a negative insertion loss value in front of the design domain and a high insertion loss value behind the design domain. However, the insertion loss for the lowest frequencies is rather small, and below $40 \mathrm{~Hz}\left(\lambda_{\mathrm{R}}=4.7 \mathrm{~m}\right)$, the insertion loss for the optimized design is lower than for the reference design. Depending on the actual loading, more weight can be given to certain frequencies by introducing a weighting function in Eq. (25).

\section{Geometric imperfections}

Topology optimization often provides designs that are only optimal for the specific problem considered. In many cases, the performance is very sensitive to geometric imperfections. Consider for example the design in figure 6a. This design contains small features, making it very sensitive to deviations in the geometry of these features. As a consequence, the performance of the actual wave barrier may be far from optimal. 


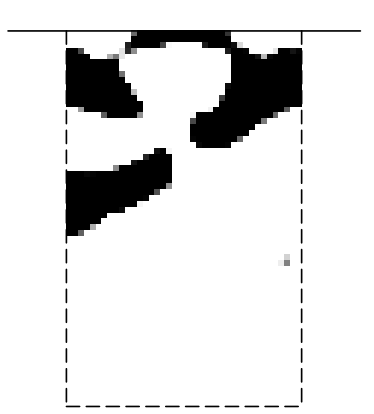

(a)

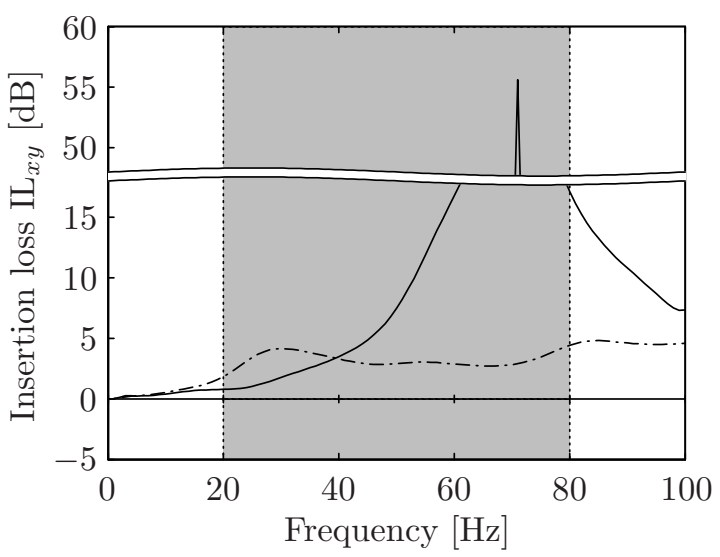

(b)

Figure 9: (a) Optimized design maximizing $\overline{\mathrm{IL}_{x y}}$ for the frequency range $20-80 \mathrm{~Hz}$ (discretized with 10 frequencies), and (b) resulting insertion loss $\mathrm{IL}_{x y}$ (Eq. (23)) as a function of the frequency for the reference design in figure 2a (dash-dotted line) and the optimized design (solid line).

The influence of errors in the (in-plane) dimensions of the stiffened material can be modeled by varying the Heaviside projection threshold $\eta$ in Eq. 13 [29]. For low values of the Heaviside projection threshold (e.g. $\eta=0.25$ ), lower values of the filtered densities are projected to the stiffened material as well, and the dimensions of the stiffened material increase, leading to so-called dilated designs. For high values of the Heaviside projection threshold (e.g. $\eta=0.75$ ), only the higher values of the filtered densities are projected to the stiffened material, and the dimensions of the stiffened material decrease, leading to so-called eroded designs.

Figures 11a-c show the dilated, intermediate, and eroded version of the design optimized for a frequency of $25 \mathrm{~Hz}$ (figure 4). The corresponding insertion loss values are indicated in figure 11d, which shows the insertion loss as a function of the projection threshold. Increasing the Heaviside projection threshold $\eta$ results in a reduction of the dimensions of the stiffened material. This is accompanied by a decrease in the insertion loss. The upper value $\eta=1$ removes all stiffened material from the design $\left(\hat{\mathbf{u}}=\hat{\mathbf{u}}_{\text {orig }}\right)$, and the insertion loss (Eq. (16)) becomes equal to zero.

Figure 12 shows the influence of the Heaviside projection threshold $\eta$ for the design optimized for reducing transmission at a frequency of $50 \mathrm{~Hz}$ (figure 6). In contrast to the design optimized for a frequency of $25 \mathrm{~Hz}$, a high value occurs at $\eta=0.5$ (intermediate design). The performance is therefore very sensitive to thickness variations as these disturb the previously discussed interference (see section 3). For the dilated design $(\eta=0.25)$, a high performance is still obtained thanks to the top part of the design, which is largely affected for the eroded design $(\eta=0.75)$.

The effect of the projection threshold on the designs maximizing the minimal and frequency averaged insertion loss in the frequency range $20-80 \mathrm{~Hz}$ is shown in figures 13 and 14 . These results show that the design maximizing the minimal insertion loss (Eq. (26)) is less sensitive to thickness variations than the design maximizing the frequency averaged insertion loss (Eq. (24)). The former is significantly affected only for values of $\eta$ above 0.6 , leading to modifications in the top part of the design which result in a lower performance at higher frequencies.

In order to obtain designs which are less sensitive to this type of geometric imperfections, a robust topology optimization approach is adopted. Here, robust topology optimization is applied to account for deviations from the design geometry maximizing the frequency averaged insertion loss. The interval of the projection threshold is set to $[0.25,0.75]$.

Since the insertion loss in figure 14 does not show any sharp variations, a worst case formulation $[15,14]$ considering only three cases is expected to give good results. The robust optimization problem is formulated 


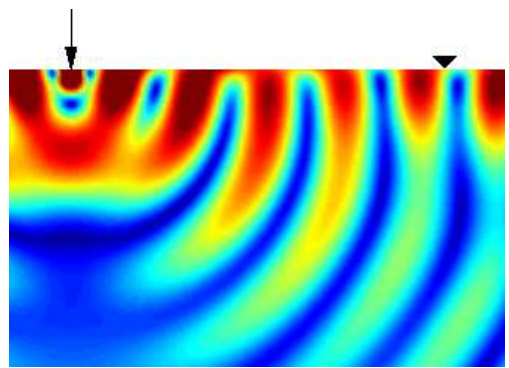

(a)

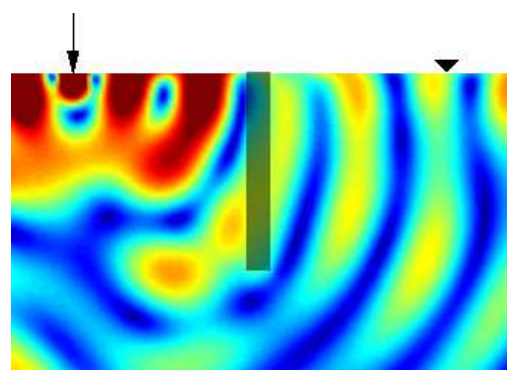

(d)

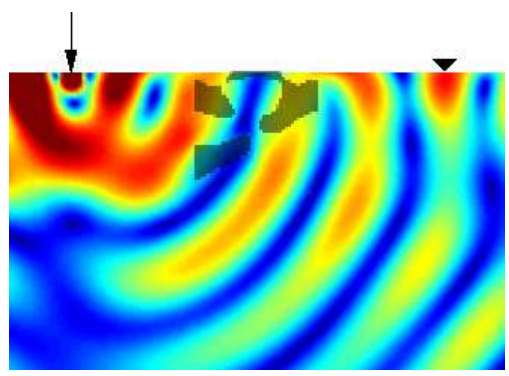

(g)

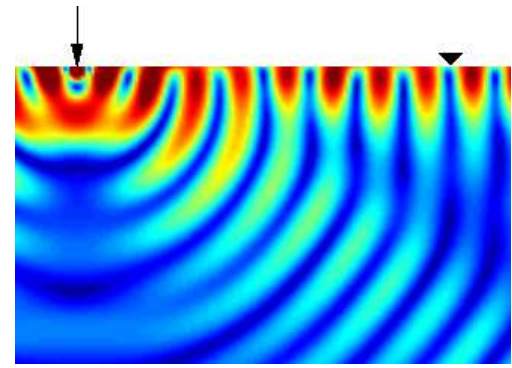

(b)

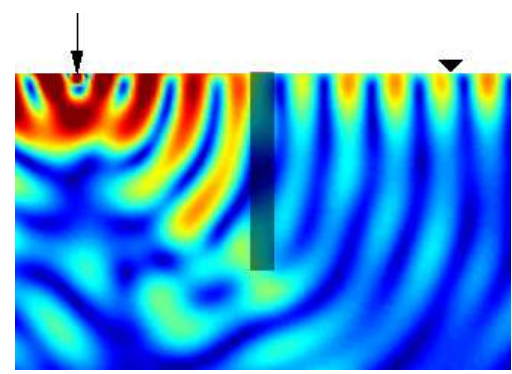

(e)

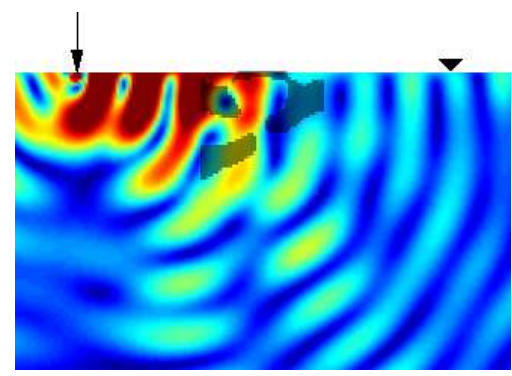

(h)

$\hat{\mathbf{u}}^{R}[\mu \mathrm{m} / \mathrm{kN}$

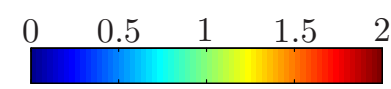

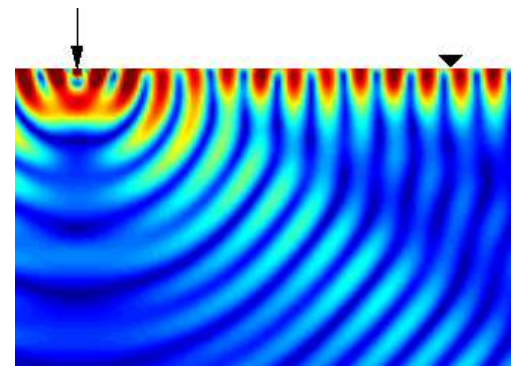

(c)

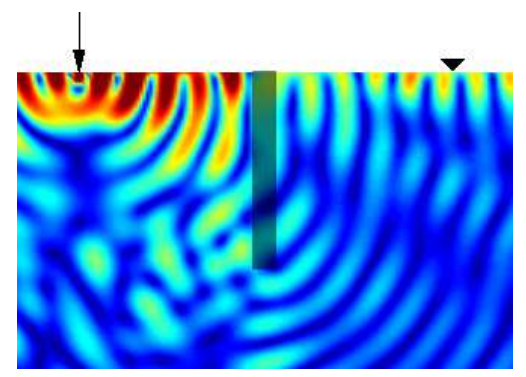

(f)

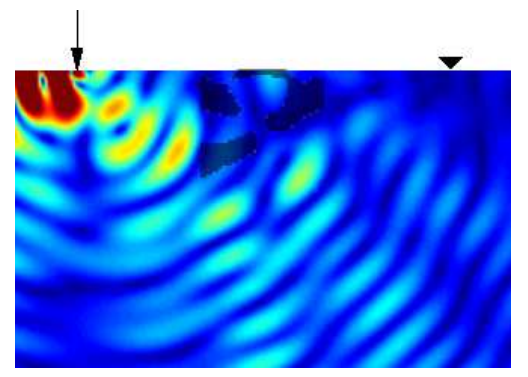

(i)

Figure 10: Real part of the displacement field $\hat{\mathbf{u}}^{R}$ in the homogeneous halfspace for excitation at (a) $30 \mathrm{~Hz}$, (b) $50 \mathrm{~Hz}$, and (c) $70 \mathrm{~Hz}$, with the reference design for excitation at (d) $30 \mathrm{~Hz}$, (e) $50 \mathrm{~Hz}$, and (f) $70 \mathrm{~Hz}$, and with the design maximizing the frequency averaged insertion loss $\overline{\mathrm{IL}_{x y}}$ for excitation at (g) $30 \mathrm{~Hz},(\mathrm{~h}) 50 \mathrm{~Hz}$, and (i) $70 \mathrm{~Hz}$.

as follows:

$$
\begin{aligned}
\min _{\rho_{e}} & \max \left\{\Phi^{\prime}\left(\bar{\rho}_{e}^{d}\right), \Phi^{\prime}\left(\bar{\rho}_{e}^{i}\right), \Phi^{\prime}\left(\bar{\rho}_{e}^{e}\right)\right\} \\
\text { s.t. } & \sum_{e=1}^{N} v_{e} \bar{\rho}_{e}^{i} \leq V^{*}, e=1 \ldots N \\
& 0 \leq \rho_{e} \leq 1, e=1 \ldots N \\
\text { with } \quad & {\left[\begin{array}{cc}
\hat{\mathbf{K}}\left(\bar{\rho}_{e}^{d}\right) & \mathbf{Q}^{T} \\
\mathbf{Q} & \mathbf{0}
\end{array}\right]\left\{\begin{array}{l}
\hat{\mathbf{u}}^{d} \\
\chi^{d}
\end{array}\right\}=\left\{\begin{array}{l}
\hat{\mathbf{p}} \\
\mathbf{0}
\end{array}\right\} } \\
& {\left[\begin{array}{cc}
\hat{\mathbf{K}}\left(\bar{\rho}_{e}^{i}\right) & \mathbf{Q}^{T} \\
\mathbf{Q} & \mathbf{0}
\end{array}\right]\left\{\begin{array}{l}
\hat{\mathbf{u}}^{i} \\
\chi^{i}
\end{array}\right\}=\left\{\begin{array}{l}
\hat{\mathbf{p}} \\
\mathbf{0}
\end{array}\right\} } \\
& {\left[\begin{array}{cc}
\hat{\mathbf{K}}\left(\bar{\rho}_{e}^{e}\right) & \mathbf{Q}^{T} \\
\mathbf{Q} & \mathbf{0}
\end{array}\right]\left\{\begin{array}{l}
\hat{\mathbf{u}}^{e} \\
\chi^{e}
\end{array}\right\}=\left\{\begin{array}{l}
\hat{\mathbf{p}} \\
\mathbf{0}
\end{array}\right\} }
\end{aligned}
$$




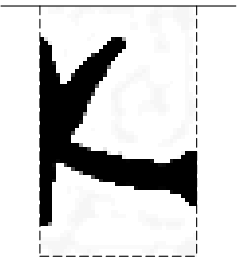

(a)

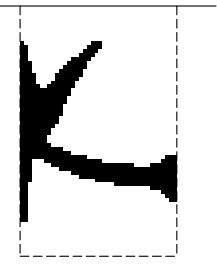

(b)

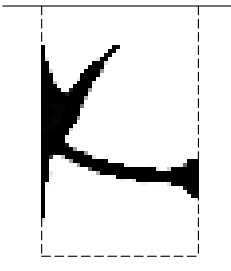

(c)

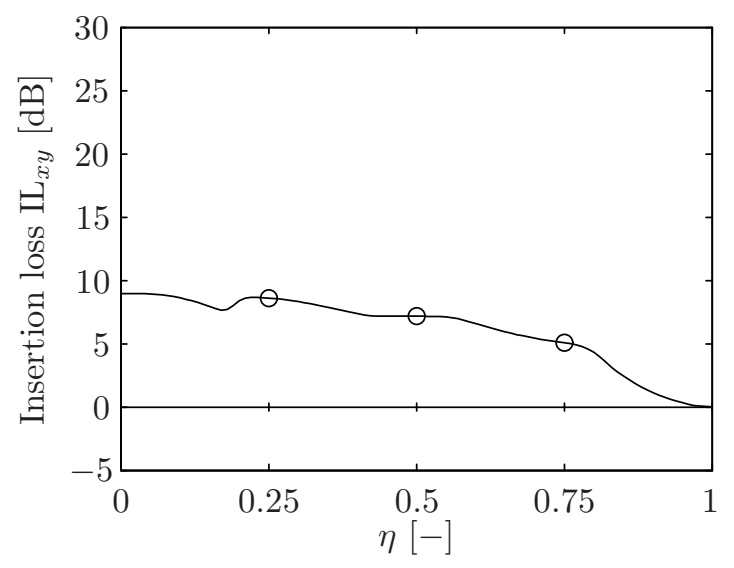

(d)

Figure 11: The (a) dilated $(\eta=0.25)$, (b) intermediate $(\eta=0.5)$, and (c) eroded $(\eta=0.75)$ version of the optimized design maximizing the insertion loss $\mathrm{IL}_{x y}$ at a frequency of $25 \mathrm{~Hz}$ (figure $4 \mathrm{a}$ ) and (d) the influence of the projection threshold $\eta$ on the insertion loss $\mathrm{IL}_{x y}$ at $25 \mathrm{~Hz}$.

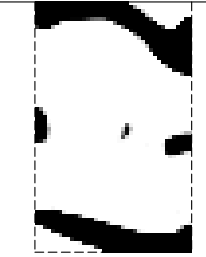

(a)

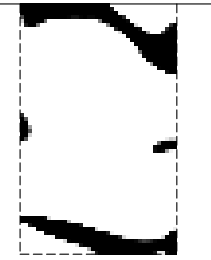

(b)

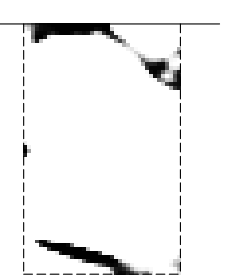

(c)

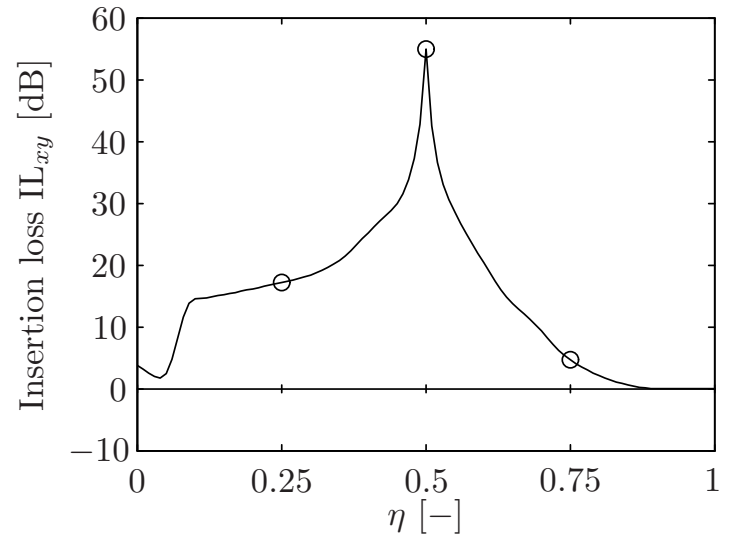

(d)

Figure 12: The (a) dilated $(\eta=0.25)$, (b) intermediate $(\eta=0.5)$, and (c) eroded $(\eta=0.75)$ version of the optimized design maximizing the insertion loss $\mathrm{IL}_{x y}$ at a frequency of $50 \mathrm{~Hz}$ (figure 6a) and (d) the influence of the projection threshold $\eta$ on the insertion loss $\mathrm{IL}_{x y}$ at $50 \mathrm{~Hz}$.

where $\bar{\rho}_{e}^{d}, \bar{\rho}_{e}^{i}$, and $\bar{\rho}_{e}^{e}$ are the dilated $\left(\eta=\eta_{0}-\Delta \eta\right)$, intermediate $\left(\eta=\eta_{0}\right)$ and eroded $\left(\eta=\eta_{0}+\Delta \eta\right)$ volume densities, respectively, with corresponding stiffness matrices $\hat{\mathbf{K}}\left(\bar{\rho}_{e}^{d}\right), \hat{\mathbf{K}}\left(\bar{\rho}_{e}^{i}\right)$, and $\hat{\mathbf{K}}\left(\bar{\rho}_{e}^{e}\right)$, corresponding displacements $\hat{\mathbf{u}}^{d}, \hat{\mathbf{u}}^{i}$, and $\hat{\mathbf{u}}^{e}$, and corresponding Lagrange multipliers $\chi^{d}, \chi^{i}$, and $\chi^{e}$.

As before, the dilated design corresponds to a value of the Heaviside projection threshold equal to $\eta=0.25$, the intermediate design to a value $\eta=0.5$, and the eroded design to a value $\eta=0.75$. Figure 15 shows the resulting robust design and insertion loss. The design is rather similar to the deterministic design in figure $9 \mathrm{a}$ and also results in a peak value of the insertion loss at the higher frequencies of the range considered. The thin connection in the left upper part and the small inclusion in the lower right part of the deterministic design (figures 9a) have disappeared, however. The changes in geometry lead to a slightly lower (deterministic) performance for the intermediate design, resulting in a frequency averaged insertion loss of $13.1 \mathrm{~dB}$ instead of $14.2 \mathrm{~dB}$.

The robust design is less sensitive to thickness variations, as can be seen in figure 16, where the frequency 


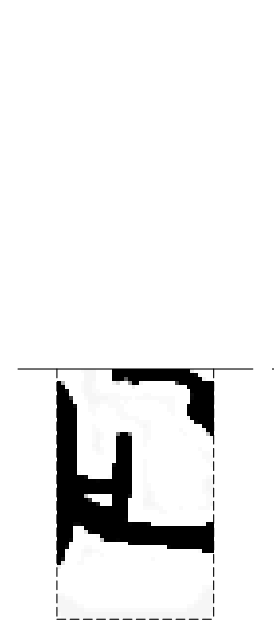

(a)

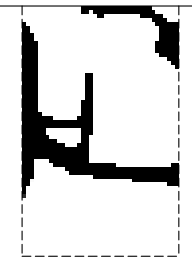

(b)

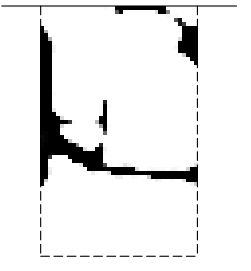

(c)

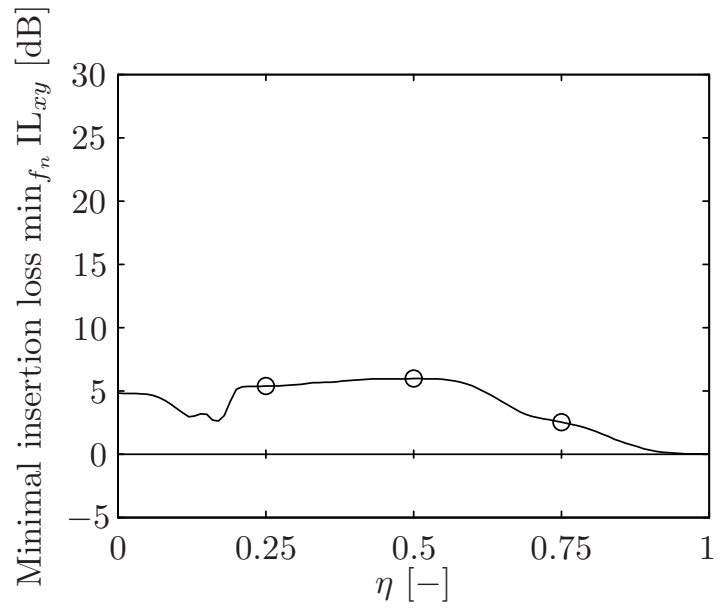

(d)

Figure 13: The (a) dilated $(\eta=0.25)$, (b) intermediate $(\eta=0.5)$, and (c) eroded $(\eta=0.75)$ version of the optimized design maximizing the minimal insertion loss $\min _{f_{n}} \mathrm{IL}_{x y}\left(f_{n}\right)$ for the frequency range $20-80 \mathrm{~Hz}$ (figure 8a) and (d) the influence of the projection threshold $\eta$ on the minimal insertion loss $\min _{f_{n}} \mathrm{IL}_{x y}$ in the frequency range $20-80 \mathrm{~Hz}$.

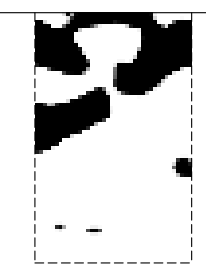

(a)

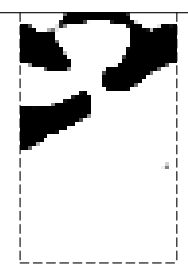

(b)

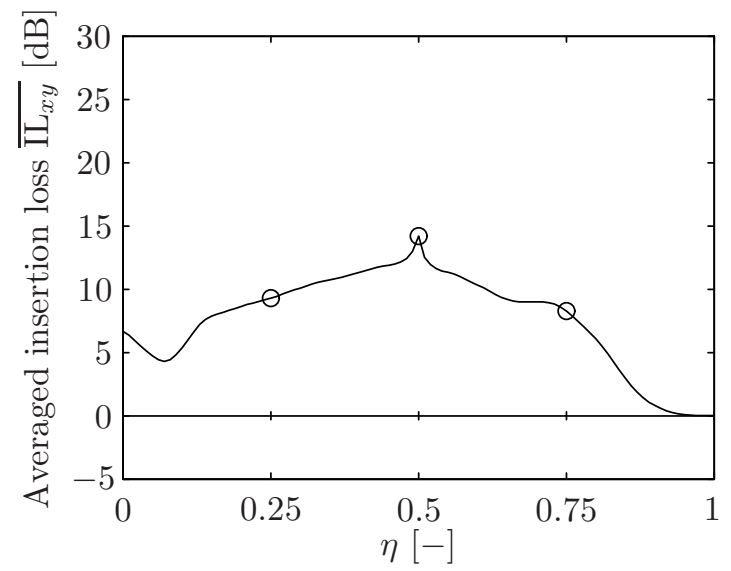

(d)

Figure 14: The (a) dilated $(\eta=0.25)$, (b) intermediate $(\eta=0.5)$, and (c) eroded $(\eta=0.75)$ version of the optimized design maximizing the frequency averaged insertion loss $\overline{\mathrm{IL}_{x y}}$ for the frequency range $20-80 \mathrm{~Hz}$ (figure 9a) and (d) the influence of the projection threshold $\eta$ on the frequency averaged insertion loss $\overline{\mathrm{IL}_{x y}}$ in the frequency range $20-80 \mathrm{~Hz}$.

averaged insertion loss $\overline{\mathrm{IL}_{x y}}$ is plotted as a function of the projection threshold $\eta$. There are some small peaks at $\eta=0.25, \eta=0.5$, and $\eta=0.75$, but these are not as distinct as the peak at $\eta=0.5$ in figure 14 . The frequency averaged insertion loss is therefore larger than $11.0 \mathrm{~dB}$ over the entire range $\eta=[0.25,0.75]$.

Because of manufacturing constraints, it may be difficult to reproduce the design in figure 15 . Since the design is robust to thickness variation, a simplification of the geometry is not expected to significantly affect the performance of the design. Figure 17 shows a manually simplified design and the corresponding insertion loss $\mathrm{IL}_{x y}$ by intuitively positioning three parallelograms where the stiffened material is concentrated in the topology optimized design. Comparing figures $15 \mathrm{~b}$ and $17 \mathrm{~b}$ shows that the performance of the latter design is similar to the performance of the design obtained with robust topology optimization. The peak value of the insertion loss at higher frequencies is lower, and the frequency averaged insertion loss is equal to $9.1 \mathrm{~dB}$ instead of $13.1 \mathrm{~dB}$, but the robust design still outperforms the reference design which has a frequency averaged insertion loss of $3.2 \mathrm{~dB}$. 


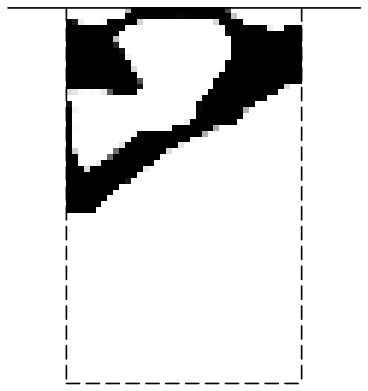

(a)

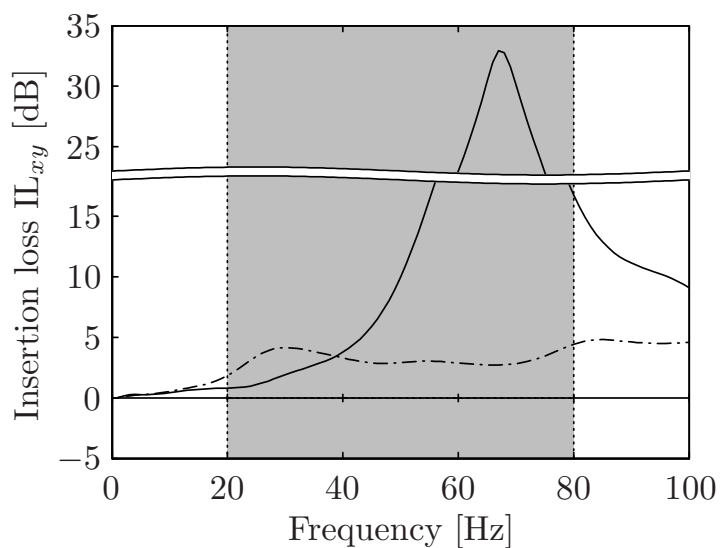

(b)

Figure 15: (a) Optimized design maximizing $\overline{\mathrm{IL}_{x y}}$ for the frequency range $20-80 \mathrm{~Hz}$ (discretized with 10 frequencies) using a worst case robust approach, and (b) resulting insertion loss $\mathrm{IL}_{x y}$ (Eq. (23)) as a function of the frequency for the reference design in figure $2 \mathrm{a}$ (dash-dotted line) and the optimized design (solid line).

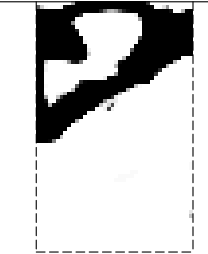

(a)

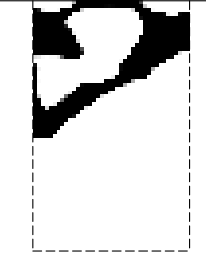

(b)

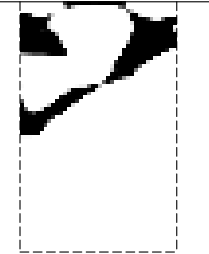

(c)

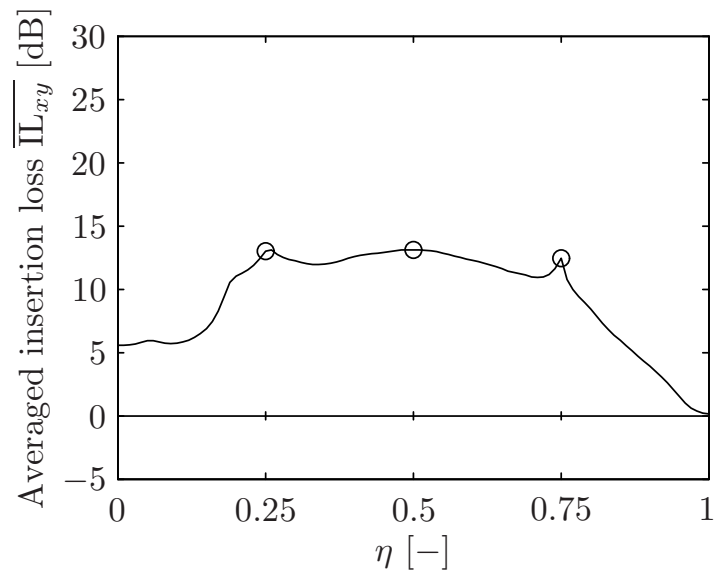

(d)

Figure 16: The (a) dilated $(\eta=0.25)$, (b) intermediate $(\eta=0.5)$, and (c) eroded $(\eta=0.75)$ version of the robust optimized design maximizing the frequency averaged insertion loss $\overline{\mathrm{IL}} x y$ for the frequency range $20-80 \mathrm{~Hz}$ (figure $15 \mathrm{a}$ ) and (d) the influence of the projection threshold $\eta$ on the frequency averaged insertion loss $\overline{\mathrm{IL}_{x y}}$ in the frequency range $20-80 \mathrm{~Hz}$.

\section{Conclusion}

In this paper, topology optimization is used to design wave barriers for the reduction of elastodynamic wave transmission. An elastic halfspace is modeled using finite elements, and a PML technique is adopted to avoid spurious wave reflections at the boundaries. Topology optimization is used to minimize wave transmission by optimally distributing a stiffer material in a design domain. The insertion loss is used to quantify the effectiveness of the mitigation measure and is maximized at an output point.

Three cases are considered. First, a harmonic source at a fixed frequency is considered, resulting in a distribution of the stiffened material that optimally reflects and guides waves away from the surface. At higher frequencies, high values of the insertion loss are obtained due to interference patterns. Second, for harmonic sources at a frequency in a given range, the minimal insertion loss is maximized, leading to a uniform increase of the insertion loss over the frequency range of interest. Third, in the case where the frequency averaged insertion loss is maximized, the resulting optimized design mainly reduces the insertion 


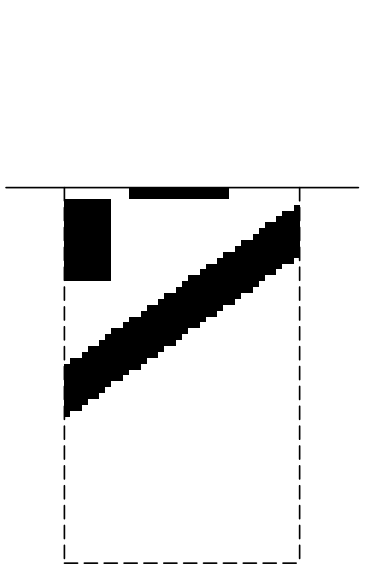

(a)

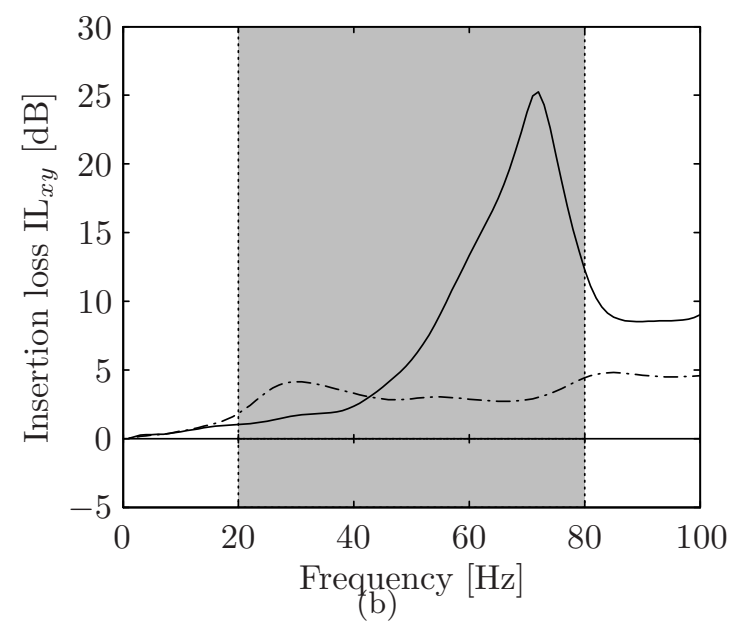

Figure 17: (a) Simplified design after a manual post-processing of the optimized design maximizing $\overline{\mathrm{IL}_{x y}}$ for the frequency range $20-80 \mathrm{~Hz}$ (discretized with 10 frequencies) using a worst case robust approach (figure 15a), and (b) resulting insertion loss $\mathrm{IL}_{x y}$ (Eq. (23)) as a function of the frequency for the reference design in figure 2a (dash-dotted line) and the post-processed optimized design (solid line).

loss at the highest frequencies, where it is easier to achieve a high insertion loss.

Geometric imperfections can have an important influence on the performance of designs obtained by topology optimization. The design optimized for the frequency averaged insertion loss is found to be more sensitive to deviations in design geometry than the one optimized for the minimal insertion loss. A worst case robust approach is applied to improve the former design. The use of the robust approach makes it possible to simplify the topology with little deterioration of performance. The resulting design outperforms the classical rectangular wave barrier.

\section{Acknowledgments}

The first author is a doctoral fellow of the Research Foundation Flanders (FWO). The financial support is gratefully acknowledged. The work was partly carried out during a research stay at the DTU, supported by Villum Fonden through the NextTop project and the Research Foundation Flanders (FWO). The first, third, and last author are members of the KU Leuven-BOF PFV/10/002 OPTEC-Optimization in Engineering Center. The authors would also like to thank Stine Skov Kristensen since initial steps and inspiration to this work partly originate from her Master's thesis.

\section{A. Derivation of the sensitivities}

For the derivation of the sensitivities, the objective function $\Phi=10 \log \left(\frac{\hat{\mathbf{u}}^{H} \mathbf{L} \hat{\mathbf{u}}}{\hat{\mathbf{u}}_{\text {orig }}^{H} \mathbf{L} \hat{\mathbf{u}}_{\text {orig }}}\right)$ is extended by the conditions of the equilibrium equation given in Eq. (12) (multiplied by the vectors $\boldsymbol{\lambda}$ and $\boldsymbol{\mu}$ ):

$$
\Phi^{\text {adj }}=\Phi+\boldsymbol{\lambda}^{T}\left(\hat{\mathbf{K}} \hat{\mathbf{u}}+\mathbf{Q}^{T} \boldsymbol{\chi}-\hat{\mathbf{p}}\right)+\boldsymbol{\lambda}^{H}\left(\hat{\mathbf{K}}^{*} \hat{\mathbf{u}}^{*}+\mathbf{Q}^{T} \boldsymbol{\chi}^{*}-\hat{\mathbf{p}}^{*}\right)+\boldsymbol{\mu}^{T} \mathbf{Q} \hat{\mathbf{u}}+\boldsymbol{\mu}^{H} \mathbf{Q} \hat{\mathbf{u}}^{*}
$$


where a star denotes the complex conjugate. The derivatives of the objective function with respect to the design variable are then computed as:

$$
\begin{aligned}
\frac{\partial \Phi^{\mathrm{adj}}}{\partial \bar{\rho}_{e}}= & \frac{\partial \Phi}{\partial \hat{\mathbf{u}}^{R}} \frac{\partial \hat{\mathbf{u}}^{R}}{\partial \bar{\rho}_{e}}+\frac{\partial \Phi}{\partial \hat{\mathbf{u}}^{I}} \frac{\partial \hat{\mathbf{u}}^{I}}{\partial \bar{\rho}_{e}}+\boldsymbol{\lambda}^{T}\left(\frac{\partial \hat{\mathbf{K}}}{\partial \bar{\rho}_{e}} \hat{\mathbf{u}}+\hat{\mathbf{K}} \frac{\partial \hat{\mathbf{u}}}{\partial \bar{\rho}_{e}}+\mathbf{Q}^{T} \frac{\partial \boldsymbol{\chi}}{\partial \bar{\rho}_{e}}\right) \\
& +\boldsymbol{\lambda}^{H}\left(\frac{\partial \hat{\mathbf{K}}^{*}}{\partial \bar{\rho}_{e}} \hat{\mathbf{u}}^{*}+\hat{\mathbf{K}}^{*} \frac{\partial \hat{\mathbf{u}}^{*}}{\partial \bar{\rho}_{e}}+\mathbf{Q}^{T} \frac{\partial \boldsymbol{\chi}^{*}}{\partial \bar{\rho}_{e}}\right)+\boldsymbol{\mu}^{T} \mathbf{Q} \frac{\partial \hat{\mathbf{u}}}{\partial \bar{\rho}_{e}}+\boldsymbol{\mu}^{H} \mathbf{Q} \frac{\partial \hat{\mathbf{u}}^{*}}{\partial \bar{\rho}_{e}}
\end{aligned}
$$

In this equation, the displacement vectors are decomposed in a real and imaginary part: $\hat{\mathbf{u}}=\hat{\mathbf{u}}^{R}+\mathrm{i} \hat{\mathbf{u}}^{I}$ and $\hat{\mathbf{u}}^{*}=\hat{\mathbf{u}}^{R}-\mathrm{i} \hat{\mathbf{u}}^{I}$. Rearranging gives:

$$
\begin{aligned}
\frac{\partial \Phi^{\text {adj }}}{\partial \bar{\rho}_{e}}= & \boldsymbol{\lambda}^{T} \frac{\partial \hat{\mathbf{K}}}{\partial \bar{\rho}_{e}} \hat{\mathbf{u}}+\boldsymbol{\lambda}^{H} \frac{\partial \hat{\mathbf{K}}^{*}}{\partial \bar{\rho}_{e}} \hat{\mathbf{u}}^{*} \\
& +\left(\frac{\partial \Phi}{\partial \hat{\mathbf{u}}^{R}}+\boldsymbol{\lambda}^{T} \hat{\mathbf{K}}+\boldsymbol{\lambda}^{H} \hat{\mathbf{K}}^{*}+\boldsymbol{\mu}^{T} \mathbf{Q}+\boldsymbol{\mu}^{H} \mathbf{Q}\right) \frac{\partial \hat{\mathbf{u}}^{R}}{\partial \bar{\rho}_{e}} \\
& +\left(\frac{\partial \Phi}{\partial \hat{\mathbf{u}}^{I}}+\mathrm{i} \boldsymbol{\lambda}^{T} \hat{\mathbf{K}}-\mathrm{i} \boldsymbol{\lambda}^{H} \hat{\mathbf{K}}^{*}+\mathrm{i} \boldsymbol{\mu}^{T} \mathbf{Q}-\mathrm{i} \boldsymbol{\mu}^{H} \mathbf{Q}\right) \frac{\partial \hat{\mathbf{u}}^{I}}{\partial \bar{\rho}_{e}} \\
& +\left(\boldsymbol{\lambda}^{T} \mathbf{Q}^{T}+\boldsymbol{\lambda}^{H} \mathbf{Q}^{T}\right) \frac{\partial \boldsymbol{\chi}^{R}}{\partial \bar{\rho}_{e}}+\left(\mathrm{i} \boldsymbol{\lambda}^{T} \mathbf{Q}^{T}-\mathrm{i} \boldsymbol{\lambda}^{H} \mathbf{Q}^{T}\right) \frac{\partial \boldsymbol{\chi}^{I}}{\partial \bar{\rho}_{e}}
\end{aligned}
$$

In order to eliminate the expressions involving the derivatives of $\hat{\mathbf{u}}$ and $\boldsymbol{\chi}$, the bracketed terms must be equal to zero:

$$
\begin{gathered}
\boldsymbol{\lambda}^{T} \hat{\mathbf{K}}+\boldsymbol{\lambda}^{H} \hat{\mathbf{K}}^{*}+\boldsymbol{\mu}^{T} \mathbf{Q}+\boldsymbol{\mu}^{H} \mathbf{Q}=-\frac{\partial \Phi}{\partial \hat{\mathbf{u}}^{R}} \\
\mathrm{i} \boldsymbol{\lambda}^{T} \hat{\mathbf{K}}-\mathrm{i} \boldsymbol{\lambda}^{H} \hat{\mathbf{K}}^{*}+\mathrm{i} \boldsymbol{\mu}^{T} \mathbf{Q}-\mathrm{i} \boldsymbol{\mu}^{H} \mathbf{Q}=-\frac{\partial \Phi}{\partial \hat{\mathbf{u}}^{I}} \\
\boldsymbol{\lambda}^{T} \mathbf{Q}^{T}+\boldsymbol{\lambda}^{H} \mathbf{Q}^{T}=\mathbf{0} \\
\mathrm{i} \boldsymbol{\lambda}^{T} \mathbf{Q}^{T}-\mathrm{i} \boldsymbol{\lambda}^{H} \mathbf{Q}^{T}=\mathbf{0}
\end{gathered}
$$

This leads to the following system of equations:

$$
\left[\begin{array}{cc}
\hat{\mathbf{K}} & \mathbf{Q}^{T} \\
\mathbf{Q} & \mathbf{0}
\end{array}\right]\left\{\begin{array}{l}
\boldsymbol{\lambda} \\
\boldsymbol{\mu}
\end{array}\right\}=\left\{\begin{array}{c}
-\frac{1}{2}\left(\frac{\partial \Phi}{\partial \hat{\mathbf{u}}^{R}}-\mathrm{i} \frac{\partial \Phi}{\partial \hat{\mathbf{u}}^{I}}\right)^{T} \\
\mathbf{0}
\end{array}\right\}
$$

If $\boldsymbol{\lambda}$ and $\boldsymbol{\mu}$ satisfy this adjoint equation, the sensitivities in Eq. (A.3) become:

$$
\frac{\partial \Phi^{\mathrm{adj}}}{\partial \bar{\rho}_{e}}=2 \operatorname{Re}\left\{\lambda^{T} \frac{\partial \hat{\mathbf{K}}}{\partial \bar{\rho}_{e}} \hat{\mathbf{u}}\right\}
$$

If $\hat{\mathbf{u}}$ and $\chi$ satisfy Eq. (12), this means that $\Phi^{\text {adj }}=\Phi$, implying that the expressions on the right hand side of Eq. (A.9) provide the sensitivities of the actual objective function $\Phi$.

\section{References}

[1] M. Bendsøe, O. Sigmund, Topology optimization: theory, methods and applications, Springer, Berlin, 2003.

[2] C. Rupp, M. Frenzel, A. Evgrafov, K. Maute, M. Dunn, Design of nanostructured phononic materials, in: Proceedings of ASME 2005 International Mechanical Engineering Congress and Exposition, American Society of Mechanical Engineers, Orlando, Florida, USA, 2005, pp. 483-488.

[3] P. Borel, A. Harpøth, L. Frandsen, M. Kristensen, P. Shi, J. Jensen, O. Sigmund, Topology optimization and fabrication of photonic crystal structures, Optics Express 12 (9) (2004) 1996-2001. 
[4] J. Jensen, O. Sigmund, Topology optimization of photonic crystal structures: a high-bandwidth low-loss T-junction waveguide, Journal of the Optical Society of America B 22 (6) (2005) 1191-1198.

[5] O. Sigmund, J.S. Jensen, Systematic design of phononic band-gap materials and structures by topology optimization, Philosophical Transactions of the Royal Society of London Series A: Mathematical, Physical and Engineering Sciences 361 (1806) (2003) 1001-1019.

[6] C. Van hoorickx, M. Schevenels, G. Lombaert, Topology optimization of one-dimensional wave impeding barriers, in: P. Sas, D. Moens, H. Denayer (Eds.), Proceedings of ISMA 2014 International Conference on Noise and Vibration Engineering, Leuven, Belgium, 2014, pp. 3557-3571.

[7] J.S. Jensen, Topology optimization problems for reflection and dissipation of elastic waves, Journal of Sound and Vibration 301(2007) 319-340.

[8] E. Wadbro, M. Berggren, Topology optimization of an acoustic horn, Computer Methods in Applied Mechanics and Engineering 196 (2006) 420-436.

[9] M. Dühring, J. Jensen, O. Sigmund, Acoustic design by topology optimization, Journal of Sound and Vibration 317 (3) (2008) $557-575$

[10] D. Greiner, J. Aznárez, O. Maeso, G. Winter, Single-and multi-objective shape design of Y-noise barriers using evolutionary computation and boundary elements, Advances in Engineering Software 41 (2) (2010) 368-378.

[11] K. Abe, T. Fujiu, K. Koro, Shape optimization with topological derivative and its application to noise barrier for railway viaducts, in: C. Zhang, M. Aliabadi, M. Schanz (Eds.), Proceedings of the 11th International Conference of Advances in Boundary Element Techniques XI, EC Ltd, 2010, pp. 7-12.

[12] J. Kook, K. Koo, J. Hyun, J. Jensen, S. Wang, Acoustical topology optimization for Zwickers loudness model - Application to noise barriers, Computer Methods in Applied Mechanics and Engineering 237 (2012) 130-151.

[13] M. Jansen, G. Lombaert, M. Schevenels, Robust topology optimization of structures with imperfect geometry based on geometric nonlinear analysis, Computer Methods in Applied Mechanics and Engineering 285 (2015) $452-467$.

[14] O. Sigmund, Manufacturing tolerant topology optimization, Acta Mechanica Sinica 25 (2) (2009) $227-239$.

[15] F. Wang, B. Lazarov, O. Sigmund, On projection methods, convergence and robust formulations in topology optimization, Structural and Multidisciplinary Optimization 43 (6) (2011) 767-784.

16] P. Coulier, S. François, G. Degrande, G. Lombaert, Subgrade stiffening next to the track as a wave impeding barrier for railway induced vibrations, Soil Dynamics and Earthquake Engineering 48 (2013) 119-131.

[17] J. Lysmer, R. Kuhlemeyer, Finite dynamic model for infinite media, Journal of the Engineering Mechanics Division 95 (EM4) (1969) 859-877.

[18] J. Bérenger, A perfectly matched layer for the absorption of electromagnetic waves, Journal of Computational Physics 41 (2) (1994) 185-200.

[19] M. Bouchon, F. Sánchez-Sesma, Boundary integral equations and boundary elements methods in elastodynamics, Advances in Geophysics 48 (2007) 157-189.

[20] I. Harari, U. Albocher, Studies of FE/PML for exterior problems of time-harmonic elastic waves, Computer Methods in Applied Mechanics and Engineering 195 (29) (2006) 3854-3879.

[21] S. Kristensen, Topology optimization for damping of ground vibration, Master's thesis, DTU (2012).

[22] R. Cook, D. Malkus, M. Plesha, R. Witt, Concepts and applications of finite element analysis, 4th Edition, John Wiley \& Sons, 2002.

[23] K. Svanberg, The method of moving asymptotes - a new method for structural optimization, International Journal for Numerical Methods in Engineering 24 (2) (1987) 359-373.

[24] J.K. Guest, J.H. Prévost, T. Belytschko, Achieving minimum length scale in topology optimization using nodal design variables and projection functions, International Journal for Numerical Methods in Engineering 61 (2) (2004) 238-254

[25] M. Bendsøe, Optimal shape design as a material distribution problem, Structural optimization 1 (4) (1989) 193-202.

[26] G. Rozvany, M. Zhou, T. Birker, Generalized shape optimization without homogenization, Structural optimization 4 (3-4) (1992) 250-252.

[27] S. François, M. Schevenels, B. Thyssen, J. Borgions, G. Degrande, Design and efficiency of a vibration isolating screen in the soil, Soil Dynamics and Earthquake Engineering 39 (2012) 113-127.

[28] K. Svanberg, Some modelling aspects for the Matlab implementation of MMA (2004).

[29] M. Schevenels, B. Lazarov, O. Sigmund, Robust topology optimization accounting for spatially varying manufacturing errors, Computer Methods in Applied Mechanics and Engineering 200 (49) (2011) 3613-3627. 